\title{
Warped Radion Dark Matter
}

\author{
Aníbal D. Medina ${ }^{a}$ and Eduardo Pontón ${ }^{b}$ \\ ${ }^{a}$ Department of Physics, University of California, One Shields Ave. Davis, CA $95616^{a}$, USA \\ ${ }^{c}$ Department of Physics, Columbia University, 538 W. 120th St, New York, NY 10027, USA
}

October 17, 2018

\begin{abstract}
Warped scenarios offer an appealing solution to the hierarchy problem. We consider a non-trivial deformation of the basic Randall-Sundrum framework that has a KK-parity symmetry. This leads to a stable particle beyond the Standard Model, that is generically expected to be the first KK-parity odd excitation of the radion field. We consider the viability of the KK-radion as a DM candidate in the context of thermal and non-thermal production in the early universe. In the thermal case, the KK-radion can account for the observed DM density when the radion decay constant is in the natural multi- $\mathrm{TeV}$ range. We also explore the effects of coannihilations with the first KK excitation of the RH top, as well as the effects of radion-Higgs mixing, which imply mixing between the KK-radion and a KK-Higgs (both being KK-parity odd). The non-thermal scenario, with a high radion decay constant, can also lead to a viable scenario provided the reheat temperature and the radion decay constant take appropriate values, although the reheat temperature should not be much higher than the TeV scale. Direct detection is found to be feasible if the DM has a small (KK-parity odd) Higgs admixture. Indirect detection via a photon signal from the galactic center is an interesting possibility, while the positron and neutrino fluxes from KK-radion annihilations are expected to be rather small. Colliders can probe characteristic aspects of the DM sector of warped scenarios with KK-parity, such as the degeneracy between the radion and the KK-radion (DM) modes.
\end{abstract}




\section{Introduction}

Models of electroweak symmetry breaking (EWSB) that contain a stable particle closely tied to the EWSB sector open a window of complementarity between astrophysical observations, DM searches, and collider experiments. Not surprisingly, such a prospect has attracted considerable attention in the context of supersymmetry, little Higgs models with T-parity [1] or models with Universal Extra Dimensions (UED) [2].

The latter case is interesting in that the reason the DM candidate is stable is closely tied to the geometry/compactification of the extra dimensional space [3, 4]. In the 5D case, this is simply due to a $Z_{2}$ reflection symmetry (usually called KK-parity) about the center of the compactified fifth dimension. The viability of the lightest KK-parity odd particle (the LKP) as a dark matter (DM) candidate was first studied in [5, 6]. For a review, see [7]. An important assumption in the previous construction is that the extra dimensional spacetime is flat. On the other hand, since the seminal work of [8], the presence of a non-negligible spacetime curvature (although with vanishing 4D effective cosmological constant), has been understood to have rather important and interesting consequences. Unfortunately, in typical implementations, no stable new particles appear in such warped scenarios, although it is simple to build models with a stable DM candidate by simply imposing a $Z_{2}$ symmetry (see e.g. 9]). There have also been proposals where the DM and EWSB sectors share nontrivial properties [10, 11, 12], within the framework of warped compactifications, as well as studies in "warped throats" that appear in type IIB string theory on Calabi-Yau manifolds where a suitable DM candidate can be obtained [13]. Also, Ref. [14] has considered the possible Sommerfeld enhancements arising from radion exchange for GUT-motivated DM candidates in warped scenarios, while stable candidates arising from a dark sector in warped compactifications have been proposed in Ref. [15].

It is nevertheless possible, and not difficult, to build warped scenarios with a KK-parity symmetry, similar to UED models. The first attempt was done in [16], where it was proposed to glue together two copies of the RS model along either the UV or IR branes. This reference considered the possibility that the first KK-parity odd excitation of the $Z$-gauge boson could be the DM candidate. Nevertheless, it is well known that the lightest state beyond the SM in such constructions is expected to be the radion field [17]. If this field has a KK-parity odd excitation, it is more likely that this "KK-radion" will be the LKP. A concrete model that stabilizes the interbrane distance, and hence gives mass to the radion field, while at the same time leading to the existence of a KK-parity symmetry, was proposed in Ref. [18] (see [19] for an alternative proposal). Indeed, it is found that generically the LKP is the first KK-radion mode.

In this work, we explore the viability of the KK-radion as a DM candidate. Given that the radion is such an intrinsic feature of extra-dimensional scenarios, having it constitute most of the matter density of the universe would be a rather pleasing possibility. The KKradion mass is found to be parametrically lighter than the scale of the typical Kaluza-Klein (KK) resonances, which is set by $\tilde{k}_{\text {eff }} \equiv k_{\text {eff }} e^{-A(L)}$, where $k_{\text {eff }}$ is the curvature at the (IR) boundaries of the metric background $d s^{2}=e^{-2 A(y)} \eta_{\mu \nu} d x^{\mu} d x^{\nu}-d y^{2}$. In models where the KK scale is of order TeV, so that they can be tested at the LHC, the LKP mass is expected to be around the EW scale. Like the radion field itself, it couples to the SM particles (and to other KK excitations) via higher-dimension operators, suppressed by the "radion decay 
constant" [18]

$$
\Lambda_{r}=\sqrt{\frac{3 M_{5}^{3}}{k_{\text {eff }}^{3}}} \times \tilde{k}_{\mathrm{eff}}
$$

where $M_{5}$ is the 5D (reduced) Planck mass. We note that, from a theoretical point of view, $\Lambda_{r}$ can take on a large range of values by varying the ratio $M_{5} / k_{\text {eff }}$, while keeping $\tilde{k}_{\text {eff }} \sim \mathrm{TeV}$. In fact, it was pointed out in Ref. [18] that varying the ratio $M_{5} / k_{\text {eff }}$ allows to interpolate between RS-like "strong warping" scenarios (when $k_{\text {eff }} \sim M_{5}$ ) and "UED-like" scenarios (when $k_{\text {eff }} \ll M_{5}$ ). Although the former case may be considered more natural, we will also consider the second possibility, since it gives an interesting deformation of UED models, that shares properties of pure UED and the RS model. Furthermore, this is also an interesting limit from the point of view of the KK-radion relic density.

As is well-known, obtaining the correct relic abundance of an EW scale thermal relic requires that its interactions be of typical EW size. Thus, unless $\Lambda_{r}$ is sufficiently close to the EW scale, the KK-radion interactions would be too weak, the KK-radion would freeze-out rather early in the history of the universe, and the relic density following from the thermal freeze-out paradigm would be too large and lead to the "overclosure" of the universe. As we will show, however, the scenario is nevertheless viable over a wide range of $\Lambda_{r}$. First, it turns out that the self-annihilation cross section for KK-radions can be sufficiently large even when $\Lambda_{r}$ is in the multi-TeV range. Second, the presence of other relatively light colored resonances can lead to a sufficiently strong depletion of KK-radions through coannihilation effects, and make the scenario viable for $\Lambda_{r}$ as large as $\mathcal{O}\left(10^{4} \mathrm{TeV}\right)$. Mixing with a $\mathrm{KK}$ excitation of the Higgs can have a similar effect. For much larger values of the radion decay constant, it is possible to imagine that the KK-radions never reached thermal equilibrium in the early universe. This is the case in the "UED-like" (or "small-warping") scenario mentioned above. It is then necessary that the non-thermal production of KK-radions be sufficiently suppressed. This restricts the range of allowed $\Lambda_{r}$ for a given reheat temperature, $T_{R}$. For instance, if $T_{R} \sim 1 \mathrm{TeV}$, one must have $\Lambda_{r} \sim 10^{15} \mathrm{GeV}$.

The outline of this paper is as follows. In Section 2, we summarize the relevant properties of the scenario proposed in Ref. [18. In Section 3, we comment on the expected finite temperature properties of our setup. In Section 4, we consider the KK-radion relic density in the thermal freeze-out scenario, while we consider the effects of coannihilations in Section 5 . The case of non-thermal production and the related constraints are treated in Section 6 . We also comment on the prospects for direct and indirect DM searches for KK-radion DM, as well as on the collider signals that can help in distinguishing this scenario from other possibilities. We conclude in Section 8. We provide two appendices where we discuss the KK-radion couplings and the effects of EWSB (Appendix A), and where we summarize the relevant cross sections and decay rates (Appendix B).

\section{Models with Warped KK-Parity}

We start by summarizing the salient properties of the scenario presented in Ref. [18. This is a $5 \mathrm{D}$ model with the fifth dimension compactified to an interval, and parameterized by 
$y \in[-L, L]$. The size of the extra dimension is stabilized at tree-level by a bulk (GW) scalar field, $\Phi$, similar to the Goldberger-Wise proposal [17]. However, it is assumed that the gravitational backreaction of the stabilizing scalar is non-negligible, and that it is the main source for a non-trivial warping of the 5D spacetime. When the scalar potential satisfies $V(-\Phi)=V(\Phi)$, and allows for a kink-like background solution $\langle\Phi(y)\rangle=\phi(y)$, with $\phi(-y)=-\phi(y)$, the associated warp factor, $e^{-A(y)}$, is found to be even about $y=0$, i.e. one finds $A(-y)=A(y)$. The non-trivial kink configuration is assumed to be chosen over the trivial vacuum expectation value (VEV), $\phi(y)=0$, by appropriate potentials on the infrared (IR) boundaries, at $y= \pm L$. The background scalar energy density is concentrated around $y=0$, thus leading to a dynamically generated "UV brane" at the origin. In general, this is a "fat" brane with thickness governed by the mass parameter appearing in the bulk scalar potential, although there is a well defined limit where the UV brane is thin and the background has a constant, negative curvature. In this limit, one recovers the IR-UV-IR configuration considered in Ref. [16].

For the purpose of the present work, the most important consequence of the above setup is the existence of a discrete $Z_{2}$ symmetry (KK-parity), under which the KK modes whose wavefunctions are even about $y=0$ are assigned KK-parity +1 , while those whose wavefunctions are odd about $y=0$ are assigned KK-parity -1 . The resulting KK-parity symmetry is a good quantum number, and the lightest KK-parity odd excitation (the LKP) is exactly stable.

It is also important to note that a generic feature of the spectrum is the relative degeneracy between the KK-parity even and KK-parity odd states of a given 5D field. The reason is that what differentiates even from odd states are the properties of the corresponding wavefunctions, $f_{n}(y)$, at the origin: KK-parity even states satisfy $f_{n}^{\prime}(0)=0$, while KK-parity odd states satisfy $f_{n}(0)=0$. But when these wavefunctions are localized near the IR boundaries, so that their values are (sometimes exponentially) suppressed at the origin, only a small deformation is required to convert an even wavefunction into an odd one. Therefore, the associated KK masses are very close. This observation is particularly interesting when applied to IR localized (would-be) zero-modes. We highlight three important examples.

First, the lightest physical scalar excitation of the 5D metric/bulk scalar system is a KK-parity even field that is typically referred to as the "radion". Its wavefunction is given by $F_{0}(y) \approx e^{2[A(y)-A(L)]}$. This state has a mass that is parametrically lighter than the typical KK scale. From the argument above, the first KK-parity odd scalar excitation has a mass that is exponentially close to the radion mass (at tree-level). This "KK-radion" is expected to be the LKP, and shares properties closely connected to those of the radion field. We will refer to it either as $r^{\prime}$ of $r_{-}$.

Second, since a solution to the hierarchy problem requires the Higgs field to be highly localized near the IR boundaries, one finds light KK-parity even and KK-parity odd $S U(2)$ Higgs doublets. The KK-parity even doublet is fully responsible for EWSB (giving rise to a SM-like Higgs and the would-be Goldstone modes eaten by the $W^{ \pm}$and $Z$ ). The KK-parity odd Higgs is "inert" in the sense that it does not participate in EWSB, but it can be parametrically lighter than the KK scale since, were it not for EWSB, it would be exponentially degenerate with the KK-parity even Higgs doublet, at tree-level. However, we will assume that it is somewhat heavier than the KK-radion, since it is a generic scalar whose mass is not protected by any symmetry. Annihilation of KK-radions into the KK-parity even 
Higgs degrees of freedom will be found to play a relevant role in setting the DM relic density.

Third, fermion zero-modes can be localized either near the "UV brane" (at the center of the fifth dimension) or near the IR boundaries. An appealing possibility is to use this localization properties to understand the observed fermion mass hierarchies [20, 21]. Thus, assuming that the 5D Yukawa couplings are all of the same order, the lighter SM fermions (which include the RH tau and bottom) are localized in the UV region, while the top quark is localized closer to the IR branes. In particular, one might expect that the RH top is localized close to the IR boundaries, and therefore its first KK-parity odd excitation is expected to be the lightest of the fermionic resonances. How light this state is depends on how closely localized to the IR branes the RH top is. We will refer to this KK-parity odd particle as $t^{\prime}$. The next-to-lightest KK-parity odd particle (NLKP) is either $t^{\prime}$ or the inert Higgs doublet above.

It should be pointed out that in the anarchic scenarios, where the 5D Yukawa couplings are sufficiently large to generate the top mass, with the $(t, b) S U(2)$-doublet as close to the first two generations as possible (to minimize potentially large corrections to the $Z b_{L} \bar{b}_{L}$ coupling), the effects from EWSB are non-negligible. We take these into account by diagonalizing the KK mass matrix in the presence of the EWSB contributions. A consequence of this is that the lightest KK-parity odd fermion resonances are pushed downward in mass ("level repulsion" in the absence of a zero-mode for the KK-parity odd subtower). As a result, we find that the $t^{\prime}$ cannot be heavier than about a factor of 4 above the radion mass, and is typically much closer to $m_{r} \approx m_{r^{\prime}}$. However, one might also wish to consider non-anarchic scenarios where the Yukawa couplings themselves are hierarchical [22, 23], and EWSB effects are much less important.

To summarize, the states lighter than the KK scale, $\tilde{k}_{\text {eff }}$, are the radion, its first KK-parity odd mode (which is stable), a light KK-parity odd $t^{\prime}$ not much heavier than the previous two, and an additional Higgs doublet, on top of the SM field content. Our main objective in this work will be to study the viability of this scenario from the point of view of DM constraints. If the KK radion is indeed the LKP, these are strongly dependent on the radion decay constant given in Eq. (1), and we will explore several possibilities.

\section{High-Temperature Phase Transitions}

Before presenting the analysis of the KK-radion relic density, we comment on the expected high-temperature properties of the present scenario. In the well-studied case of the RS background with the radion stabilized via a Goldberger-Wise scalar, the transition from a hightemperature "deconfined phase" to a low-temperature "confined" one has been discussed in Refs. [24, 25, 26, 27, 28, 29, 30]. The terminology arises from the AdS/CFT correspondence [31, 32], where the phase transition maps into a deconfinement/confinement phase transition in the dual, large $N$ gauge theory (this phase transition is strongly first-order). The analysis has been performed on the gravity side, and in the limit that the backreaction of the stabilizing GW scalar is small, by comparing two finite-temperature solutions obeying the same boundary conditions on the UV brane (however, Ref. [29] considered the case where the backreaction is large). These are: the Euclidean RS background, with an IR brane and Euclidean time (as measured on the UV brane) compactified on $[0, \beta=1 / T]$; and the 
AdS-Schwarzschild (AdS-S) solution, where the IR brane is replaced by a black-hole horizon. The free energy associated with the RS solution vanishes as a result of the requirement that the effective $4 \mathrm{D}$ cosmological constant be zero at the minimum of the radion potential. The free energy of the AdS-S solution is then given by $F_{\text {dec }} \sim E_{0}-c N^{2} T^{4}$, where $E_{0} \sim m_{r}^{2} \Lambda_{r}^{2}$ is a vacuum energy contribution, and the second term corresponds to a plasma of deconfined "gluons" with $N^{2}$ degrees of freedom at temperature $T$ ( $c$ is an order one number). Recalling that under the correspondence, $\Lambda_{r} \sim N \tilde{k}$, it follows that the transition temperature is of order $T_{c} \sim\left(m_{r}^{2} \tilde{k}^{2}\right)^{1 / 4}$, where $\tilde{k}$ is the warped-down curvature scale.

Our setup differs from the above RS scenario in two ways: there is no "UV boundary", and the backreaction of the stabilizing dynamics is not a perturbation (but rather the major source of the warping). Also, the analog of the AdS-S solution is not known in this setup, although it is plausible that such a solution -with black-hole horizons symmetrically located with respect to $y=0$ - exists, and has similar properties to the one mentioned above (recall that there is a limit where our scenario reduces to two copies of RS glued together at the UV brane). By analogy, one might expect that the scaling relations of the previous paragraph also hold in the present case, with $\tilde{k} \rightarrow \tilde{k}_{\text {eff }}$. In addition, below $T_{c}$, the rate of true-vacuum bubble nucleation is expected to be suppressed by $e^{-\mathcal{O}\left(N^{2}\right)}$. For moderate values of $N$, i.e. $\Lambda_{r}$ near the KK scale, one can then expect that, as the temperature drops below $T_{c}$, there will be a period of supercooling, and an associated inflationary era while the universe remains trapped in the false vacuum, as discussed in [24, 27] for the RS case. Eventually, bubbles nucleate and the prompt decay of the radion reheats the universe to a temperature of order $\mathrm{TeV}$. From then on the history of the universe is standard, and the KK-radion relic density is set by the dynamics of states that are parametrically lighter than the KK scale, and are in thermal equilibrium. The result is unambiguous and independent of the precise reheat temperature, $T_{R}$.

However, we will also be interested in scenarios with small warping ( $k_{\text {eff }} \ll M_{5}$ ), hence $\Lambda_{r} \gg \tilde{k}_{\text {eff }}$. Since this corresponds to the very large $N$ limit, we expect that the rate of bubble nucleation will be extremely suppressed. To avoid an empty universe, we therefore need to assume that the highest reheat temperature never exceeded $T_{c}$, and that the universe was in the "confined" phase throughout its history. Note that in our scenario $m_{r} \sim \tilde{k}_{\text {eff }} / \sqrt{k_{\text {eff }} L}$ is at the EW scale for any $\Lambda_{r}$. Therefore, $T_{c}$ is expected to be at the TeV scale, and is independent of $N$. Nevertheless, to account for possible uncertainties in the above analysis that may allow $T_{c}$ to be slightly larger, we will also study, at least formally, the case in which the reheat temperature is as large as an order of magnitude above $\tilde{k}_{\text {eff }}$ (roughly the $4 \mathrm{D}$ cutoff of the KK theory). With this in mind, we proceed to describe several scenarios that can lead to KK-radion DM.

\section{The KK-Radion as a WIMP}

We first consider the $r^{\prime}$ relic density in the standard thermal freeze-out scenario. As mentioned in the introduction, all the interactions of the KK-radion, like those of the radion itself, are non-renormalizable and controlled by the radion decay constant, $\Lambda_{r}$. An important ex-

\footnotetext{
${ }^{1}$ In terms of the dual 4D picture language, the explicit breaking of the conformal symmetry is in no sense small at the scale where this symmetry is spontaneously broken.
} 


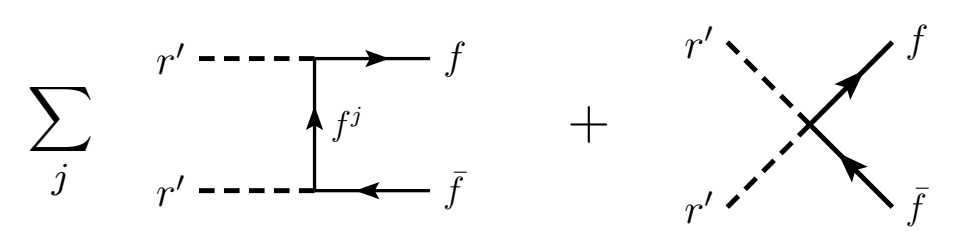

Figure 1: Self-annihilation of $r^{\prime}$ into fermion pairs. The u-channel diagram is understood.

ception arises from a possible mixing with the Higgs, and we will consider this case separately at the end of this section. It is therefore a question whether these non-renormalizable interactions are sufficiently strong to deplete the thermal $r^{\prime}$ density down to acceptable levels, which will set an upper bound on $\Lambda_{r}$. A possible loophole arises when $\Lambda_{r}$ is so large that it is possible to imagine that the KK-radions never reached thermal equilibrium in the first place. We postpone the discussion of such a scenario to Section 6. Assuming then that the $r^{\prime}$ are in thermal equilibrium at a high temperature, the self-annihilation into SM particles are responsible for maintaining a thermal number distribution when the temperature falls below the KK-radion mass, $m_{r^{\prime}}$.

The KK-radions can annihilate into fermion pairs, as shown in Fig. 1. The annihilation into massless gauge bosons are suppressed at tree-level by $\left(m_{r^{\prime}} / m_{V^{j}}\right)^{4}$, where $m_{V^{j}}$ is the mass of a gauge KK mode, as well as by a small (volume suppressed) coupling (see Eq. 68) of Appendix B.1] in Ref. [18]). We shall therefore ignore these channels in the following. The annihilation into the $W$ and $Z$ gauge bosons proceed through their longitudinal polarizations, and can be roughly taken into account through the annihilation into Higgses shown in Fig. 2 (this approximation will be sufficient for our purpose).

We give the full annihilation cross sections in the non-relativistic limit in Appendix B [Eqs. 63)-67)]. Note that, as indicated in Fig. 1, the annihilation into fermion pairs involves a sum over the fermion KK tower in the $t$ and $u$-channels. Including, for illustration, a single state $f^{j}$, the total annihilation cross section into fermion pairs can be well approximated by

$$
\left.\mathrm{v} \sigma_{r^{\prime} r^{\prime} \rightarrow f \bar{f}}\right|_{j} \sim \frac{N_{c} m_{r^{\prime}}^{2}}{4 \pi \Lambda_{r}^{4}}\left(1-\epsilon_{f, r^{\prime}}^{2}\right)^{3 / 2}\left[G_{1100} \epsilon_{f, r^{\prime}}-2 G_{1 j 0}^{2} \frac{\epsilon_{j, r^{\prime}}^{2}}{1+\epsilon_{j, r^{\prime}}^{2}-\epsilon_{f, r^{\prime}}^{2}}\left(\epsilon_{j, r^{\prime}}+\epsilon_{f, r^{\prime}}\right)\right]^{2}
$$

where $N_{c}=3\left(N_{c}=1\right)$ for quarks (leptons), $\epsilon_{f, r^{\prime}}=m_{f} / m_{r^{\prime}}, \epsilon_{j, r^{\prime}}=m_{j} / m_{r^{\prime}}$, and $G_{1 j 0}$ characterizes the $r^{\prime}-f_{j} \bar{f}$ vertex, while $G_{1100}$ characterizes the $r^{\prime} r^{\prime}-f \bar{f}$ vertex. ${ }^{2}$ It would seem, from the second term in the square brackets, that the cross section increases like $m_{j}^{2}$ for heavy KK states. However, in the same limit, the coupling constants $G_{1 j 0}$ decrease even faster, and one can check that the sum over $j$ is typically dominated by the first couple of terms. The effects from EWSB, which are sizable for the top tower in anarchic scenarios, are important in enhancing the above effective couplings. In particular, we find that for the $t \bar{t}$ channel, $G_{1100} \approx-6$ due to numerical factors characteristic of the two-KK-radion couplings [e.g. the factor of 4 in $\tilde{X}_{i_{1} i_{2} j k}^{R L}$, given after Eq. [58]], as well as due to EWSB mixing with the

\footnotetext{
${ }^{2}$ To obtain this simplified expression we took $g_{1 j 0}^{L L} \ll 0$ (assuming $t_{L}$ is nearly flat), and we also neglected $g_{1 j 0}^{R R}$ (for the top, this one is not small, but ends up giving a subdominant contribution). We further took $G_{1 j 0}^{L R} \approx G_{1 j 0}^{R L}\left(\equiv G_{1 j 0}\right)$ and defined $G_{1100} \equiv G_{1100}^{L R}=G_{1100}^{R L}$ (the notation is defined in Appendix B.
} 

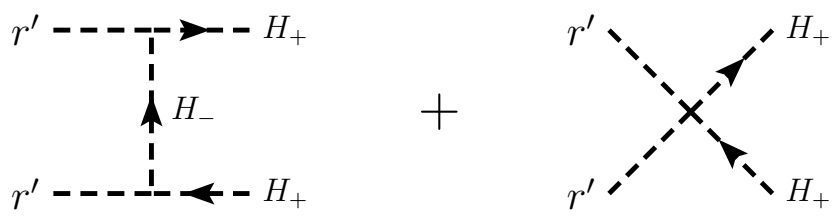

Figure 2: Self-annihilation of $r^{\prime}$ into Higgs pairs. The u-channel diagram is understood.

KK-tower. For the first top KK resonance, although $G_{110} \approx 0.3$, the enhancement due to the larger KK mass makes the second term in the square brackets of Eq. (2) non-negligible (but still subdominant). It is also important to note that $G_{1 j 0}$ is proportional to EWSB. In fact, the full expression (2) is proportional to $m_{f}^{2}$, although this fact is somewhat hidden inside $G_{1 j 0}$ for the second term in square brackets. Therefore, the annihilation rate of KK-radions into the light families is negligible compared to the annihilation into top quarks.

The annihilation of KK-radions into Higgses proceeds through the diagrams in Fig. 2 . In the limit of strong IR localization of the Higgs field, there is a KK-parity even Higgs doublet, $H_{+}$, that is responsible for EWSB, and a KK-parity odd doublet, $H_{-}$, that does not participate in EWSB, but that can be parametrically lighter than the KK scale (see comments in Section 2). We assume in this work that this KK-parity odd Higgs doublet is heavier than the KK-radion (at the end of this section we comment on the possibility that the CP-even component of the "inert" Higgs doublet is the LKP). In principle, there can be heavy Higgs resonances contributing in the $t$ and $u$-channels, as for the annihilation into fermions, but these resonances decouple in the limit that the Higgs is strongly IR localized. For simplicity, we will restrict ourselves to this limit, which will be sufficient to illustrate our point. If the SM-like Higgs is lighter than the KK-radion, while the inert Higgs doublet is heavier, we have [see Eq. (67) in Appendix B]

$$
\mathrm{v} \sigma_{r^{\prime} r^{\prime} \rightarrow H H} \sim \frac{N_{H} m_{r^{\prime}}^{2}}{\pi \Lambda_{r}^{4}}
$$

where, for large $m_{r^{\prime}}, N_{H}=4$ takes into account the SM-like Higgs as well as the would-be Goldstone modes, and therefore also annihilation into $W^{ \pm}, Z$.

In order to estimate the total KK-radion annihilation cross section, we assume that the RH top tower is localized near the IR boundaries, that the LH top is approximately uniform along the extra dimension, and that the lighter families are localized near $y=0$ (the UV region). For concreteness, and using the parameterization discussed in Ref. [18] to characterize fermion localization, which is analogous to the one used in the pure RandallSundrum $\mathrm{AdS}_{5}$ background, we take $c_{t_{R}}=-0.2$ and $c_{t_{L}}=0.52$ (these reproduce the top quark mass for $\tilde{k}_{\text {eff }} \approx 1.2 \mathrm{TeV}$, after including EWSB effects). As explained before, we can neglect annihilations into the light families, but need to take into account the annihilation into the Higgs and massive gauge bosons. For reference, in the "strong warping" benchmark scenario defined in Ref. [18] and used in this section, the branching fractions are: $\mathrm{BR}\left(r^{\prime} r^{\prime} \rightarrow\right.$ $t \bar{t}) \approx 0.70$ and $\operatorname{BR}\left(r^{\prime} r^{\prime} \rightarrow H H\right) \approx 0.30$, where $H$ denotes the four real d.o.f. in the Higgs doublet. It is expected that these are distributed as $\operatorname{BR}\left(r^{\prime} r^{\prime} \rightarrow h h\right) \approx \operatorname{BR}\left(r^{\prime} r^{\prime} \rightarrow Z Z\right) \approx$ $\frac{1}{2} \mathrm{BR}\left(r^{\prime} r^{\prime} \rightarrow W^{+} W^{-}\right) \sim 7.5 \%$. 


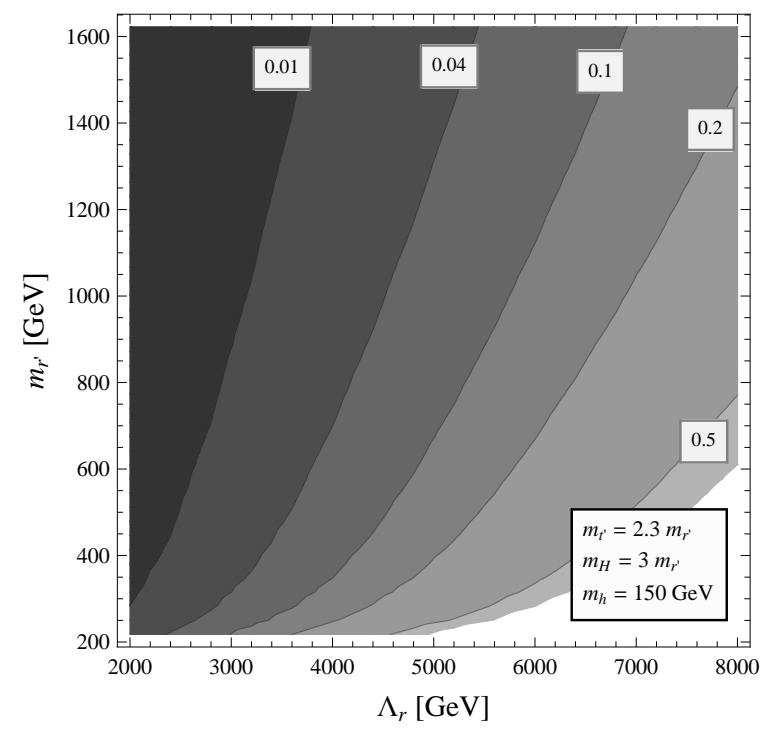

Figure 3: Contours of constant $\Omega_{r^{\prime}} h^{2}$ in the $m_{r^{\prime}}-\Lambda_{r}$ plane, in models of flavor anarchy, where EWSB effects are significant. We use the "strong warping" benchmark relation $m_{t^{\prime}} \approx$ $2.3 m_{r^{\prime}}$, so that coannihilations give a small contribution, and take a SM-like Higgs mass $m_{h} \approx 150 \mathrm{GeV}$, while the KK-parity odd doublet has mass $m_{H}=3 m_{r^{\prime}}$.

Given the total thermally averaged annihilation cross section, $\left\langle\mathrm{v} \sigma_{r^{\prime} r^{\prime}}\right\rangle$, the $r^{\prime}$ relic density is given by 33

$$
\Omega_{r^{\prime}} h^{2} \approx \frac{1.04 \times 10^{9} \mathrm{GeV}^{-1}}{M_{P l}} \frac{x_{F}}{\sqrt{g_{*}}\left\langle\mathrm{v} \sigma_{r^{\prime} r^{\prime}}\right\rangle}
$$

where $g_{*}$ is the effective number of relativistic degrees of freedom at the time of $r^{\prime}$ decoupling, $M_{P l}=1.22 \times 10^{19} \mathrm{GeV}$ is the Planck mass, and $x_{F}=m_{r^{\prime}} / T_{F}$ gives the freeze-out temperature by solving for

$$
x_{F}=\left.\log \left\{\zeta(\zeta+2) \sqrt{45 \pi}\left(\frac{g}{2 \pi^{3}}\right) \frac{m_{r^{\prime}} M_{P}\left\langle\mathrm{v} \sigma_{r^{\prime} r^{\prime}}\right\rangle}{\sqrt{g_{*} x}}\right\}\right|_{x=x_{F}}
$$

where one can take $\zeta \sim 0.5$, and in this case $g=1$.

In the left panel of Fig. 3, we show contours of constant $\Omega_{r^{\prime}} h^{2}$ in the $m_{r^{\prime}} \Lambda_{r}$ plane, including the previously described annihilation channels. Since we are assuming a flavor anarchy scenario, we take into account EWSB effects, which are non-negligible in that case. We take a SM-like Higgs mass of $m_{h}=150 \mathrm{GeV}$, while the mass of the KK-parity odd Higgs doublet is taken as $m_{H}=3 m_{r^{\prime}}$. As mentioned before, we take into account the annihilation into gauge bosons by using a multiplicative factor $N_{H}=4$ for the KK-parity even Higgs channel. We vary $m_{r^{\prime}}$ by scaling $\tilde{k}_{\text {eff }}$, i.e. keeping all mass ratios in the spectrum fixed. However, we force the SM fermions masses to remain fixed at their observed values as $\tilde{k}_{\text {eff }}$ is changed. The dimensionless couplings arising from overlap integrals are fairly insensitive to $\tilde{k}_{\text {eff }}$, and are kept fixed to the ones corresponding to the "strong warping scenario" defined in Ref. [18]. The radion decay constant can be controlled via the ratio $M_{5} / k_{\text {eff }}$, which takes 
values between about 1.0 and 2.4 in the plot. We note that in this example $m_{t^{\prime}} / m_{r^{\prime}} \approx 2.3$ with $m_{r^{\prime}} \approx 0.22 \tilde{k}_{\text {eff }}$, so that the NLKP is sufficiently heavier than $r^{\prime}$ that coannihilation effects are negligible. As can be seen from the figure, the correct relic density of $\Omega_{r^{\prime}} h^{2} \approx 0.1$ can be obtained for natural values of the parameters. In the "strong warping" benchmark scenario with $\tilde{k}_{\text {eff }}=1.2 \mathrm{TeV}$, we have $m_{r^{\prime}}=270 \mathrm{GeV}$ and $\Lambda_{r}=4.4 \mathrm{TeV}$, which leads to $\Omega_{r^{\prime}} h^{2} \approx 0.24$. Reducing $\Lambda_{r}$ to $3.6 \mathrm{TeV}$ gives agreement with the WMAP constraint. One finds $x_{F} \approx 22$, so that the freeze-out temperature is about $10 \mathrm{GeV}$.

Thus, we see that in spite of the non-renormalizable interactions of the radion, the "WIMP miracle" can be operative. This is due to sizable radion couplings to fermion pairs (associated with EWSB) and can be qualitatively understood from our expression (2). Taking $\tilde{k}_{\mathrm{eff}}=1.2 \mathrm{TeV}, m_{r^{\prime}}=0.25 \tilde{k}_{\mathrm{eff}}, m_{j}=m_{t^{\prime}} \approx 0.5 \tilde{k}_{\mathrm{eff}}, G_{1100} \approx-6$ and $G_{110} \approx 0.3$, one estimates $\left\langle\sigma_{r^{\prime} r^{\prime}} \mathrm{v} / c\right\rangle \sim 0.84 \mathrm{pb} \times\left(3 \mathrm{TeV} / \Lambda_{r}\right)^{4}$, where we recall that WMAP requires a cross section of about $0.8 \mathrm{pb}$.

One should also keep in mind that the radion interactions can be enhanced in the presence of the operator $R H^{\dagger} H$, where $R$ is the Ricci scalar. Such a term leads to kinetic mixing between the radion and CP-even, KK-parity even Higgs [34, 35], as well as between the KK radion and the $\mathrm{CP}$-even, KK-parity odd Higgs. As a result, renormalizable interactions to the SM particles are induced (although the mixing angles are formally suppressed by $v / \Lambda_{r}$ ). The presence of such a mixing offers the best chance at direct detection of KK-radion DM, and we defer the associated technical discussion to Section 7. However, here we comment on the effects for the relic density computation in the presence of such mixing. The two lightest KK-parity odd mass eigenstates are in general admixtures of $r^{\prime} \equiv r_{-}$and $h \equiv h_{-}$, which we call $\phi_{-, L}$ and $\phi_{-, H}$. The subscripts $L(H)$ refer to the lighter (heavier) of the two mass eigenstates. The DM candidate is $\phi_{\mathrm{DM}}=\phi_{-, L}$. Only when the mixing is small, or the unperturbed $m_{r^{\prime}}$ is much smaller than $m_{h_{-}}$, can this state be thought as mostly KK-radion (our assumption in the previous analysis). There is a similar mixing in the $(r, h) \equiv\left(r_{+}, h_{+}\right)$ sector, thus defining mass eigenstates $\phi_{+, L}$ and $\phi_{+, H}$. Both of these KK-parity even states contribute in the final state when they are lighter than the DM particle, although it may happen that only one is sufficiently light to contribute. The Feynman diagrams of Figs. 1 and 2 are generalized to those in Fig. 4. Note the presence of an s-channel diagram, which can lead to a resonant enhancement if any one of the KK-parity even masses is close to about twice $m_{\mathrm{DM}} \equiv m_{-, L}$. The final states include also the Goldstone modes, although those processes are not changed by the mixing with the radion tower.

The mixing with the Higgs field can significantly enhance the DM annihilation cross section, and therefore allow for larger values of $\Lambda_{r}$. As a point of reference, in the limit that $m_{h_{-}}<m_{r^{\prime}}$ and $\Lambda_{r} \rightarrow \infty$, the DM becomes pure $h_{-}$, and its annihilation cross section, which is dominated by the $t \bar{t}$ channel, reduces to

$$
\mathrm{v}{\sigma_{\phi_{D M} \phi_{D M} \rightarrow \bar{t} t}}_{2 \pi} \sim \frac{N_{c} X_{t_{R} t_{L}^{1}}^{4}}{2 \pi} \frac{m_{t^{\prime}}^{2}}{\left(m_{\mathrm{DM}}^{2}+m_{t^{\prime}}^{2}\right)^{2}},
$$

where $X_{t_{R} t_{L}^{1}}$ is the coupling of $h_{-}$to the RH top and the first KK resonance of the LH top. ${ }^{3}$ This coupling is relatively large, and therefore the result is dominated by the $t$ - (and $u-$ ) channel diagrams of the annihilation into fermions. With $X_{t_{R} t_{L}^{1}} \approx 2$ (which includes

\footnotetext{
${ }^{3}$ The third top resonance has a larger coupling that compensates for its larger mass, so that it ends up
} 

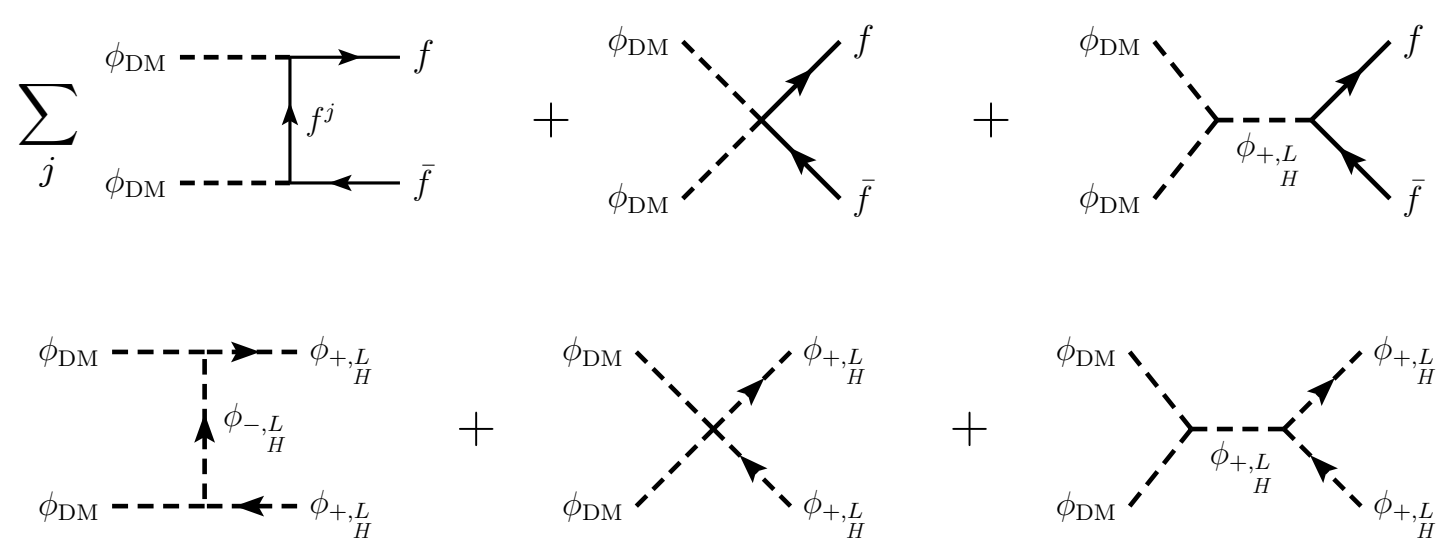

Figure 4: Self-annihilation of $\phi_{\mathrm{DM}}=\phi_{-, L}$ in the general case where the radion/Higgs and $K K$-radion/inert Higgs mix. The subscripts $L(H)$ refer to the lighter (heavier) state, while the subscripts + and - refer to KK-parity. These generalize the diagrams of Figs. 1 and 2 .

EWSB effects), and taking $m_{t^{\prime}} \sim m_{D M} \approx m_{h_{-}}$(but still assuming that $m_{t^{\prime}}>m_{h_{-}}$), we find that the observed relic density is obtained for $m_{\mathrm{DM}} \sim 30 \mathrm{TeV}$. Such large masses are not well motivated in the present scenario, and it follows that for $m_{h_{-}} \sim 1 \mathrm{TeV}$, a too large $h_{-}$component will deplete very effectively the final DM density. We conclude that the DM should be mostly KK-radion, although a small $h_{-}$component that is interesting from the point of view of direct DM searches is allowed.

\section{Coannihilations with $t^{\prime}$}

We have seen in the previous subsection that the $r^{\prime}$ self-annihilation cross section can be large enough to avoid the overproduction of KK-radions, provided the radion decay constant is sufficiently low. Does this mean that models with much larger radion decay constants are ruled out? Here we emphasize the point made in Section 2 that the first KK top excitation is expected to be relatively close to $m_{r^{\prime}}$. The reason is two-fold: first, the $S U(2)$ doublet and singlet top fields cannot be too far from the IR brane, or else $m_{t}$ cannot be reproduced. This means that the first KK-parity odd excitation of either $t_{L}$ or $t_{R}$ must be parametrically lighter than the KK scale. Second, when EWSB effects are included this state is pushed down even further due to the level repulsion of the second KK-parity odd state. 4

If it turns out that $m_{t^{\prime}}$ is relatively degenerate with the KK-radion (but heavier), coannihilations with the strongly interacting $t^{\prime}$ can become dominant. In fact, when $\Lambda_{r}$ is large,

giving a comparable contribution to the $t^{\prime}$ exchange. The heavier KK modes give a much smaller effect. The Higgs annihilation channels involve the Higgs quartic coupling, $\lambda$. For a heavy Higgs (i.e. $h_{+}$), these channels, if kinematically open, can give a contribution comparable to the $t \bar{t}$ one.

${ }^{4}$ Assuming that the 5D top Yukawa coupling saturates the NDA [36] estimate of $Y_{5 D} / L \lesssim 3 /(4 \pi)$, and taking both top zero-modes as far away as possible from the IR boundaries $\left(c_{t_{R}} \approx 0.45\right.$ and $\left.c_{t_{L}} \approx 0.48\right)$, we find that before EWSB the lightest KK-parity odd eigenvalue is about $\sim 2.31 \tilde{k}_{\text {eff }}$, which goes down to $\sim 0.89 \tilde{k}_{\text {eff }}$ when EWSB is taken into account. In the "strong warping" scenario considered in Ref. [18, with $c_{t_{R}} \approx-0.2$ and $c_{t_{L}} \approx 0.52$, we have $m_{t^{\prime}} \approx 0.5 \tilde{k}_{\text {eff }}$. The KK-radion mass is $m_{r^{\prime}} \approx 0.22 \tilde{k}_{\text {eff }}$. 

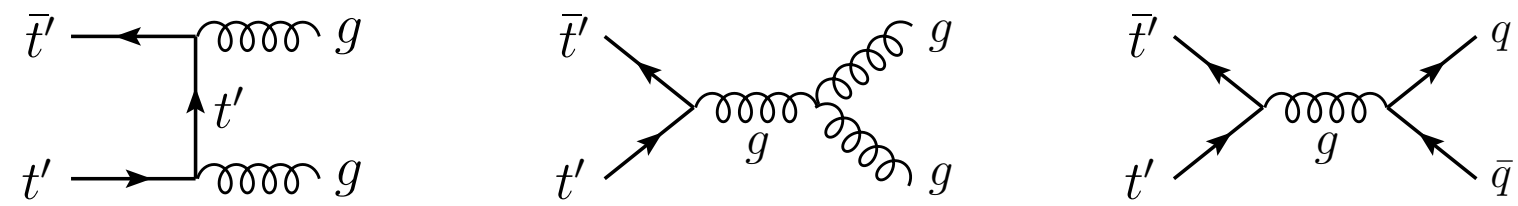

Figure 5: Self-annihilation of $t^{\prime}$ into gluon and fermion pairs via the $Q C D$ interactions.

the final $r^{\prime}$ relic density can be completely controlled by the $t^{\prime}$ self-annihilation cross section, and hence by QCD (see Fig. 5). In this case, the coannihilation channel shown in the left diagram of Fig. 6 gives a negligible contribution to the effective annihilation cross section. However, it is important that the crossed diagram, $r^{\prime} g \rightarrow \bar{t}^{\prime} t$, although suppressed by $\left(m_{t} / \Lambda_{r}\right)^{2}$, can be effective in depleting the KK-radion number density, and thus maintain the $r^{\prime}$ in equilibrium until the time the $t^{\prime}$ decouple. Indeed, for $m_{t^{\prime}} \sim m_{r^{\prime}}$ and at the temperature associated with the decoupling of $t^{\prime}$, i.e. $m_{t^{\prime}} / T_{F} \sim 25$, one has $n_{g}\left\langle\mathrm{v} \sigma\left(r^{\prime} g \rightarrow \bar{t}^{\prime} t\right)\right\rangle / n_{t^{\prime}}\left\langle\mathrm{v} \sigma\left(t^{\prime} \bar{t}^{\prime} \rightarrow g g, q \bar{q}\right)\right\rangle \sim\left(T_{F} / m_{t}\right)^{3 / 2} e^{m_{t^{\prime}} / T_{F}} \times\left(m_{t} / \Lambda_{r}\right)^{2}$. Thus, even with $\Lambda_{r}$ as large as $\sim 10^{4} \mathrm{TeV}$, the $r^{\prime}$ number density maintains an equilibrium distribution by processes such as $r^{\prime} g \rightarrow \bar{t}^{\prime} t$ (or the crossed reactions $t^{\prime} g \rightarrow r^{\prime} t$ ) until $t^{\prime}$ freeze-out.

The final relic density, after the KK tops have decayed into the KK-radions, is determined by an effective cross section [37]:

$$
\begin{aligned}
\sigma_{\mathrm{eff}}=\frac{1}{g_{\mathrm{eff}}^{2}} & {\left[\sigma_{r^{\prime} r^{\prime} \rightarrow f \bar{f}, H H}+g_{t^{\prime}} e^{-x \Delta}(1+\Delta)^{3 / 2}\left(\sigma_{r^{\prime} t^{\prime} \rightarrow g t}+\sigma_{r^{\prime} \bar{t}^{\prime} \rightarrow g \bar{t}}+\sigma_{r^{\prime} t^{\prime} \rightarrow h t}+\sigma_{r^{\prime} \bar{t}^{\prime} \rightarrow h \bar{t}}\right)\right.} \\
& \left.+g_{t^{\prime}}^{2} e^{-2 x \Delta}(1+\Delta)^{3}\left(\sum_{q} \sigma_{t^{\prime} \bar{t}^{\prime} \rightarrow q \bar{q}}+\sigma_{t^{\prime} \bar{t}^{\prime} \rightarrow g g}\right)\right], \\
g_{\mathrm{eff}}= & 1+g_{t^{\prime}} e^{-x \Delta}(1+\Delta)^{3 / 2},
\end{aligned}
$$

where $g_{t^{\prime}}=2 \times N_{c}$ is the number of degrees of freedom associated with $t^{\prime}$ (and $\bar{t}^{\prime}$ ). Here $x \equiv m_{r^{\prime}} / T$, while

$$
\Delta \equiv \frac{m_{t^{\prime}}-m_{r^{\prime}}}{m_{r^{\prime}}}
$$

parameterizes the degree of degeneracy between $r^{\prime}$ and $t^{\prime}$. Although it is straightforward to include them, we neglect EW processes involving annihilations through a $Z$ or a photon, since they give a small contribution compared to those involving gluons.

We give the expressions for $\sigma_{r^{\prime} t^{\prime} \rightarrow g t}$ and $\sigma_{r^{\prime} t^{\prime} \rightarrow h t}$ in Eqs. (69) and (70) of Appendix B. respectively. Here we focus on the case where $\Lambda_{r}$ is sufficiently large that all processes suppressed by powers of $\Lambda_{r}$ can be ignored (except, implicitly, for the purpose of maintaining thermal equilibrium, as discussed above). As a result, and provided that $t^{\prime}$ and $r^{\prime}$ are sufficiently close in mass, the final relic density is largely determined by the QCD-controlled cross sections

$$
\mathrm{v} \sigma_{t^{\prime} \bar{t}^{\prime} \rightarrow q \bar{q}} \approx \frac{2 \pi \alpha_{s}^{2}}{9 m_{t^{\prime}}^{2}}, \quad \mathrm{v} \sigma_{t^{\prime} \bar{t}^{\prime} \rightarrow g g} \approx \frac{7 \pi \alpha_{s}^{2}}{27 m_{t^{\prime}}^{2}}
$$



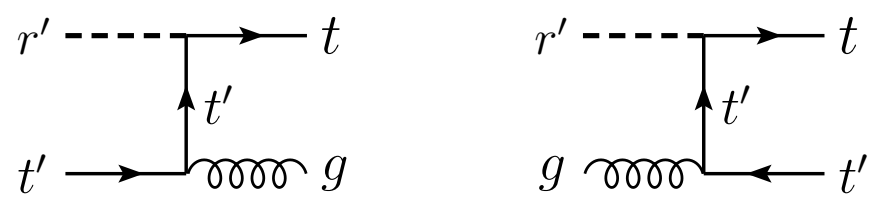

Figure 6: The left diagram (as well as a diagram where the gluon is replaced by a Higgs) can play a role in the co-annihilation scenario. The right diagram is an example of the processes responsible for maintaining the $r^{\prime}$ in thermal equilibrium until $t^{\prime}$ freeze-out.

where, for simplicity, we neglected the SM quark masses. It is straightforward to keep the full dependence on $m_{t}$, and we will do so in the numerical analysis, but the effects are relatively minor (much smaller than the errors involved in the approximation of Eq. (11) below to the full integration of the Boltzmann equations). Similarly, the velocity-dependent terms in all the cross sections make a negligible difference. In summary, we have the thermally averaged effective cross section

$$
\left\langle\mathrm{v} \sigma_{\mathrm{eff}}\right\rangle \approx \frac{172 \pi \alpha_{s}^{2}}{3 m_{t^{\prime}}^{2}} \frac{(1+\Delta)^{3}}{\left[e^{x \Delta}+6(1+\Delta)^{3 / 2}\right]^{2}} .
$$

The relic density is then approximately given by [37]

$$
\Omega_{r^{\prime}} h^{2} \approx \frac{1.04 \times 10^{9} \mathrm{GeV}^{-1}}{M_{P l}} \frac{x_{F}}{\sqrt{g_{*}} I_{a}},
$$

where $g_{*}=86.25$ is the effective number of relativistic degrees of freedom at the time of $t^{\prime}$ decoupling, the annihilations after freeze-out are taken into account by

$$
I_{a} \approx x_{F} \int_{x_{F}}^{\infty} d x \frac{\left\langle\mathrm{v} \sigma_{\mathrm{eff}}\right\rangle}{x^{2}},
$$

and $x_{F}=m_{t^{\prime}} / T_{F}$ gives the freeze-out temperature by solving Eq. (5) with the replacements $\left\langle\mathrm{v} \sigma_{r^{\prime} r^{\prime}}\right\rangle \rightarrow\left\langle\mathrm{v} \sigma_{\text {eff }}\right\rangle$ and $g \rightarrow g_{\text {eff }}$.

Imposing the 7-year WMAP constraint, $\Omega h^{2}=0.1109 \pm 0.0112$ [38], determines $\Delta$ as a function of $m_{t^{\prime}}$ (or $m_{r^{\prime}}$ ). In Fig. 7, we show this constraint, as a function of $m_{r^{\prime}}$. We see that the relic density can be accommodated for a wide range of $r^{\prime}$ masses, provided the required degeneracy with $t^{\prime}$ is present. We also show in the same figure the freeze-out temperature, as parameterized by $x_{F}$.

\section{The KK-Radion as a Non-Thermal Relic}

We now wish to consider a qualitatively different scenario: one where the KK-radion never reaches thermal equilibrium. This can happen when the radion decay constant is very large. As was explained in Section 2, this can be realized in scenarios that share properties of the Randall-Sundrum models and UED's. Hence they can be motivated outside the context of the DM relic density. We assume that the mixing with the Higgs is negligible. 


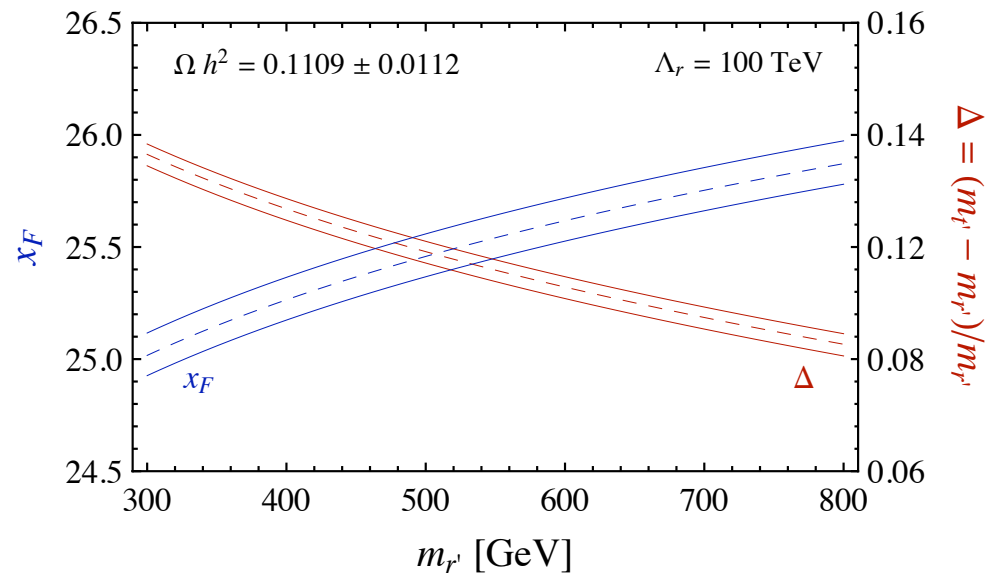

Figure 7: WMAP constraint on the degree of degeneracy, $\Delta$, as defined in Eq. (8), as a function of $m_{r^{\prime}}$ (red band, right axis). We also show the associated $x_{F}$ (blue band, left axis). We assume that $\Lambda_{r}=100 \mathrm{TeV}$, which is large enough for the relic density to be controlled by the QCD $t^{\prime}$ annihilation cross section, as well as $\Delta$.

As discussed in Section 3, when $\Lambda_{r} \gg \tilde{k}_{\text {eff }}$ we must assume that the reheat temperature is lower than the critical temperature for the deconfinement/confinement phase transition. Otherwise, the universe would effectively be trapped eternally in the false vacuum. Even if a bubble of true vacuum nucleated, the ensuing universe would be dominated by radion oscillations until their decay, which would happen rather late. The resulting reheat temperature may not be high enough to allow for a successful BBN. If instead, after a period of inflation, the inflaton decay reheats the universe only to $T_{R}<T_{c}$ we can assume that the universe was always in the "confined" phase. Due to the superweak couplings of the radion field, we can assume that it remains at its zero-temperature minimum, so that no energy is stored in the radion tower. Note also that, since the radion mass is of order the EW scale, it is easy to suppress the deSitter fluctuations of the radion field during the inflationary epoch. Thus, radion oscillations are expected to play no role, unlike in the case of UEDs with a very light radion [39]. Provided the reheat temperature is not much lower than the EW scale, KK-radions can be produced in the scatterings or decays of KK-parity odd particles in the plasma, but at a slow rate due to the suppressed couplings. Nevertheless, it is well known that such production can produce a significant number of superweakly interacting particles [40], which can translate into a bound on the reheat temperature $T_{R}$ if overclosure of the universe is to be avoided.

As we will see, this requires the reheat temperature to be relatively low, around the cutoff of the extra-dimensional theory or lower, even if we overlook the upper bound set by $T_{c}$. In addition, it bounds $\Lambda_{r}$ from below to ensure that the KK-radions are sufficiently weakly coupled. An upper bound on $\Lambda_{r}$ can also be obtained by requiring that the late decays of the NLKPs or the non-thermally produced radions do not conflict with Big-Bang nucleosynthesis $(\mathrm{BBN})$ constraints. 


\subsection{Non-Thermal Production in Scatterings and Decays}

Our point of departure is the Boltzmann equation

$$
\frac{d n_{r^{\prime}}}{d t}+3 H n_{r^{\prime}}=C
$$

where $H$ is the Hubble constant and $n_{r^{\prime}}$ is the number density of $r^{\prime}$. The collision operator on the r.h.s. contains several $r^{\prime}$ production channels that are our main concern in this section. However, since we are assuming that the $r^{\prime}$ are produced very inefficiently, so that they never reach thermal equilibrium, we neglect the inverse processes that would tend to deplete the $r^{\prime}$ number density. This assumption will be checked self-consistently at the end.

The production channels can be divided into scattering and decay processes. The former include scatterings of thermal KK fermions off the plasma, such as $g t^{j} \rightarrow r^{\prime} t^{k}$, where $t^{j}$ and $t^{k}$ are KK excitations of the top quark (including EWSB effects, these are admixtures of the doublet and singlet $S U(2)_{L}$ top towers). The towers of the other SM fermions (the "light families") can also contribute, and will be included in our analysis. In addition, at sufficiently high temperatures, the KK excitations of the gluons, $g^{n}$, can also be active and lead to $r^{\prime}$ production, e.g. via $g^{n} g^{j} \rightarrow r^{\prime} g^{k}$ or $g^{n} q^{j} \rightarrow r^{\prime} q^{k}$. Nevertheless, as we will see below, the scattering processes represent a subdominant source of thermal $r^{\prime}$ production, and therefore we will be content with obtaining an estimate for the $r^{\prime}$ production via scattering based on the processes with a zero-mode gluon and KK fermions given above. For the same reason we also do not consider scattering processes involving the weak gauge bosons $5^{5}$ We should point out that the production of higher KK resonances of the radion can also eventually produce $r^{\prime}$. However, as was emphasized in [18], the interactions of such states are controlled by a larger decay constant $\left[\Lambda_{r}^{n} \sim x_{n} \Lambda_{r}\right.$, where $m_{n}=x_{n} k_{\text {eff }} e^{-A(L)}$ is the $n$-th KK-radion mass], and therefore this contribution is negligible.

KK-radions can also be produced in (rare) decays of KK tops, $t^{j} \rightarrow r^{\prime} t^{k}$, as well as of the KK excitations of the light families, and from gauge boson decays, $V_{\mu}^{j} \rightarrow r^{\prime} V_{\mu}^{k}$, with $V_{\mu}=G_{\mu}^{a}, W_{\mu}^{ \pm}, Z_{\mu}, A_{\mu}$. We also include the decays of the inert Higgs doublet, $h_{-} \rightarrow h_{+} r^{\prime}$, $a \rightarrow G^{0} r^{\prime}$ and $H^{ \pm} \rightarrow G^{ \pm} r^{\prime}$, where $h_{+}$is the SM-like Higgs and $G^{0}, G^{ \pm}$are the would-be Nambu-Goldstone bosons eaten by the $Z$ and $W^{ \pm}$. Again, it is safe to neglect the production of heavier KK-radion resonances.

During a radiation dominated epoch the Boltzmann equation can be written in terms of $x \equiv m_{t^{\prime}} / T$ as

$$
\frac{d \tilde{n}_{r^{\prime}}}{d x}=\frac{x^{4}}{H\left(m_{t^{\prime}}\right)} C[x],
$$

where $\tilde{n}_{r^{\prime}}(x) \equiv x^{3} n_{r^{\prime}}(x)$ and $H(x)=H\left(m_{t^{\prime}}\right) x^{-2}=1.66 g_{*}^{1 / 2} m_{t^{\prime}}^{2} / M_{P l} \times x^{-2}$, with $g_{*}$ the effective number of relativistic degrees of freedom at temperature $T$. Note that we choose to measure the "time variable" $x$ in units of the $t^{\prime}$ mass, $m_{t^{\prime}}$ (we are assuming that $t^{\prime}$ is the NLKP). Eq. (14) can be integrated immediately from $x_{R}=m_{t^{\prime}} / T_{R}$, where $T_{R}$ is the reheating temperature, up to any given $x$, thus obtaining $\tilde{n}_{r^{\prime}}(x)$ and thereby $n_{r^{\prime}}(x)$.

Note that most of the heavy particles involved remain in thermal equilibrium until they disappear (by decaying) from the bath: the KK fermion resonances can decay via $t^{j} \rightarrow t^{k} V_{\mu}^{l}$,

\footnotetext{
${ }^{5}$ These are suppressed compared to the processes involving gluons by the multiplicity factor of 8 gluons versus 4 weak gauge bosons, as well as by a weak gauge coupling squared.
} 
while the KK gauge bosons can decay into fermion pairs, $V_{\mu}^{l} \rightarrow t^{j} \bar{t}^{k}$ (always preserving KK parity). These processes are controlled by renormalizable interactions and the rates are very large compared to the Hubble scale at the times of interest. The exceptions to the above are $t^{\prime}$ which must decay into $r^{\prime}$ with a rate suppressed by $\Lambda_{r}$, and the radion $\mathrm{KK}$ states whose interactions are also controlled by $\Lambda_{r}$. Of these $r^{\prime}$ is stable and by assumption remains out of thermal equilibrium. Since, as we will see, this assumption requires that $\Lambda_{r}$ be very large, the $t^{\prime}$ are quasi-stable, and freeze-out when their self-annihilation cross section becomes ineffective at $x_{F}=m_{t^{\prime}} / T_{F}$. By this time, the number density of all the heavier KK particles is highly Boltzmann suppressed, and any $r^{\prime}$ from decays of the few remaining heavy particles give a negligible contribution. Therefore, we can integrate the Boltzmann equation including all processes until the time of $t^{\prime}$ freeze-out, assuming thermal equilibrium distributions. This will give a $r^{\prime}$ number density that we will call $n_{r^{\prime}}^{(1)}$. At a somewhat later time, the remaining $t^{\prime}$ will decay out of equilibrium, giving an additional contribution to the $r^{\prime}$ number density. Assuming that there is no significant entropy production between the time of $t^{\prime}$ freeze-out and when the $t^{\prime}$ decay into $r^{\prime}$, we can effectively include this contribution at $x=x_{F}$ by an additive term $n_{r^{\prime}}^{(2)}=n_{t^{\prime}}\left(x_{F}\right)$.

The total $r^{\prime}$ relic density today is then given by $\Omega_{r^{\prime}}=m_{r^{\prime}} n_{r^{\prime}}\left(x_{0}\right) / \rho_{c}$ where $\rho_{c}=1.05 \times$ $10^{-5} h^{2} \mathrm{GeV} \mathrm{cm}{ }^{-3}=8.06 \times 10^{-47} h^{2} \mathrm{GeV}^{4}$ is the critical density of the universe today, $x_{0}$ corresponds to the temperature of the universe today, and $n_{r^{\prime}}\left(x_{0}\right)$ can be connected to $n_{r^{\prime}}\left(x_{F}\right)=n_{r^{\prime}}^{(1)}+n_{r^{\prime}}^{(2)}$ from conservation of entropy. Thus,

$$
\Omega_{r^{\prime}}^{(1)}=\frac{m_{r^{\prime}}}{\rho_{c}} \frac{s_{0}}{s\left(x_{F}\right)} \frac{1}{x_{F}^{3}} \frac{1}{H\left(m_{t^{\prime}}\right)} \int_{x_{R}}^{x_{F}} d x x^{4} C[x],
$$

where the entropy is given by

$$
s(x)=\frac{2 \pi^{2}}{45} g_{*}(x) \frac{m_{t^{\prime}}^{3}}{x^{3}},
$$

and $s_{0}=2889.2 \mathrm{~cm}^{-3} \approx 2.22 \times 10^{-38} \mathrm{GeV}^{3}$ is the entropy density today. Since $T_{F} \sim m_{t^{\prime}} / 30$ will be at most $\mathcal{O}(100 \mathrm{GeV})$, the relativistic degrees of freedom are those of the SM, e.g. $g_{*}(T=100 \mathrm{GeV})=92.25$.

The contribution coming from the decays of $t^{\prime}$ after freeze-out, as in the SWIMP scenario [41, 42], takes the form [see Eq. (4)]

$$
\Omega_{r^{\prime}}^{(2)} h^{2}=\frac{m_{r^{\prime}}}{m_{t^{\prime}}} \times \frac{1.04 \times 10^{9} \mathrm{GeV}^{-1}}{M_{P l}} \frac{x_{F}}{\sqrt{g_{*}}} \frac{1}{\left\langle\mathrm{v} \sigma_{t^{\prime} \bar{t}^{\prime} \rightarrow q \bar{q}, g g}\right\rangle},
$$

where $\mathrm{v} \sigma_{t^{\prime} \bar{t}^{\prime} \rightarrow q \bar{q}, g g}$ is determined by the QCD interactions of $t^{\prime}$, as given in Eq. (9) (see also Fig. 5). Neglecting all the quark masses (even the top quark mass gives a small correction for typical parameters), we have

$$
\left\langle\mathrm{v} \sigma_{t^{\prime} \bar{t}^{\prime} \rightarrow q \bar{q}, g g}\right\rangle \approx \frac{43}{27} \frac{\pi \alpha_{s}}{m_{t^{\prime}}^{2}}
$$

where $43 / 27=7 / 27+(2 / 9) N_{f}$, with $N_{f}=6$. The subleading corrections in the relative velocity, v, give also a negligible effect. The freeze-out temperature, parameterized by $x_{F}$, is given by Eq. (5) with $\left\langle\mathrm{v} \sigma_{r^{\prime} r^{\prime}}\right\rangle \rightarrow\left\langle\mathrm{v} \sigma_{t^{\prime} \bar{t}^{\prime} \rightarrow q \bar{q}, g g}\right\rangle, g=2 \times N_{c}=6$, and $m_{r^{\prime}} \rightarrow m_{t^{\prime}}$. 

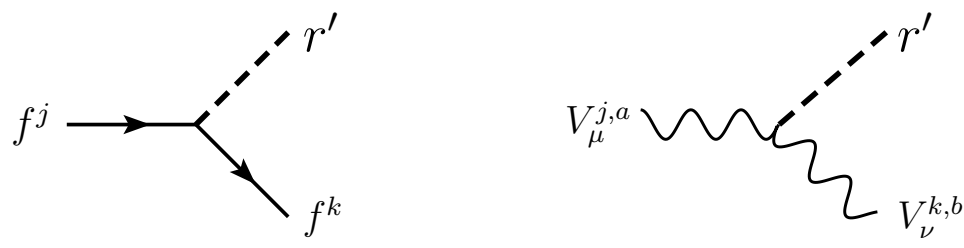

Figure 8: Production of $r^{\prime}$ in the decays of (KK-parity odd) KK fermions and KK gauge bosons. Reversing the fermion arrows gives the production from anti-fermion decays.

\subsubsection{Decay Processes Before $t^{\prime}$ Freeze-out}

We now discuss the different contributions to the collision operator in Eq. (15), starting with the production of $r^{\prime}$ in the decays of KK-parity odd fermions and gauge bosons (see Fig. 8). Using decays of KK quarks (which includes the top) for illustration, the contribution to the collision operator, per spin and color degree of freedom of the $q^{j}$, is

$$
C_{j, k}^{\text {Decay }} \equiv \int e^{-E_{q^{j}} / T} \Gamma_{q^{j} \rightarrow r^{\prime} q^{k}} \frac{d^{3} p_{q^{j}}}{(2 \pi)^{3}},
$$

where the decay rate is

$$
\Gamma_{q^{j} \rightarrow r^{\prime} q^{k}}=\frac{1}{2 E_{q^{j}}} \int(2 \pi)^{4} \delta\left(p_{q^{j}}-p_{r^{\prime}}-p_{q^{k}}\right) \overline{\left|\mathcal{M}_{q^{j} \rightarrow r^{\prime} q^{k}}\right|^{2}} \frac{d^{3} p_{r^{\prime}}}{(2 \pi)^{3} 2 E_{r^{\prime}}} \frac{d^{3} p_{q^{k}}}{(2 \pi)^{3} 2 E_{q^{k}}},
$$

which is related to the decay rate in the CM via the time dilation factor $\gamma=E_{q^{j}} / m_{j}$ by $\Gamma_{q^{j} \rightarrow r^{\prime} q^{k}}=\Gamma_{q^{j} \rightarrow r^{\prime} q^{k}}^{\mathrm{CM}} / \gamma$. Since the CM partial decay width is simply a constant, we can do the integral in Eq. (19) to obtain

$$
\begin{aligned}
C_{j, k}^{\text {decay }}\left[x_{j}\right] & =\frac{4 \pi}{(2 \pi)^{3}} m_{j}^{3} \Gamma_{j, k}^{\mathrm{CM}} \frac{K_{1}\left(x_{j}\right)}{x_{j}} \\
& \approx \frac{4 \pi}{(2 \pi)^{3}} m_{j}^{3} \Gamma_{j, k}^{\mathrm{CM}} \times\left\{\begin{array}{ll}
\sqrt{\frac{\pi}{2}} x_{j}^{-3 / 2} e^{-x_{j}} & x_{j} \gtrsim 1 \\
x_{j}^{-2} & x_{j} \ll 1
\end{array},\right.
\end{aligned}
$$

where $K_{1}(x)$ is a Bessel function, $x_{j} \equiv m_{j} / T=\left(m_{j} / m_{t^{\prime}}\right) x$, and now $\Gamma_{j, k}^{\mathrm{CM}}$ stands for any of $\Gamma_{f^{j} \rightarrow r^{\prime} f^{k}}^{\mathrm{CM}}, \Gamma_{V_{\mu}^{j} \rightarrow r^{\prime} V_{\mu}^{k}}^{\mathrm{CM}}, \Gamma_{h_{-} \rightarrow h_{+} r^{\prime}}^{\mathrm{CM}}, \Gamma_{a \rightarrow G^{0} r^{\prime}}^{\mathrm{CM}}$ or $\Gamma_{H^{ \pm} \rightarrow G^{ \pm} r^{\prime}}^{\mathrm{CM}}$, according to the case (we give their expressions in Eqs. (71), (72), (76) and (77) of Appendix B). We see that when $T_{R}<m_{j}$ (i.e. $x_{j}>1$ for all relevant times), the contribution from $C_{j, k}^{\text {decay }}$ to the r.h.s. of Eq. 15 is exponentially small. If, on the other hand, $T_{R} \gg m_{j}$, and taking into account that $m_{j} \geq m_{t^{\prime}} \gg T_{F}$, the relevant integral in Eq. (15) gives

$$
\int_{x_{R}}^{x_{F}} d x x^{4} \frac{K_{1}\left(x_{j}\right)}{x_{j}}=\epsilon_{t^{\prime}, j}^{5} \int_{m_{j} / T_{R}}^{m_{j} / T_{F}} d x_{j} x_{j}^{4} \frac{K_{1}\left(x_{j}\right)}{x_{j}} \approx \epsilon_{t^{\prime}, j}^{5}\left[\frac{3 \pi}{2}-\frac{m_{j}^{3}}{3 T_{R}^{3}}\right],
$$

where $\epsilon_{t^{\prime}, j} \equiv m_{t^{\prime}} / m_{j}$, and therefore the contribution from decays is rather insensitive to the reheat temperature in this case. The support of the integral is $1 / 2 \lesssim x_{j} \lesssim 7$, and we see 


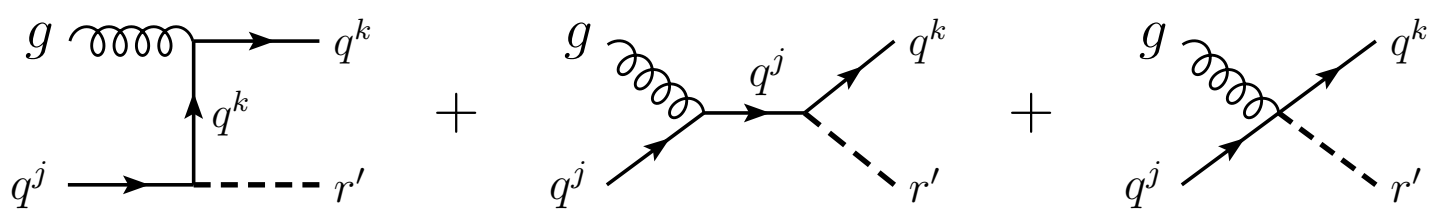

Figure 9: Tree-level scattering diagrams for $g q^{j} \rightarrow r^{\prime} q^{k}$. Reversing the fermion arrows one obtains the diagrams for $g \bar{q}^{j} \rightarrow r^{\prime} \bar{q}^{k}$. The contact diagram is required by gauge invariance.

that the bulk of $r^{\prime}$ produced in decays of heavy particles occurs for temperatures of order the mass of the decaying particle, $m_{j}$. In particular, states with masses much heavier than the reheat temperature give only an exponentially small contribution. Note also that, since parametrically $\Gamma_{j, k}^{\mathrm{CM}} \sim m_{j}^{3} / \Lambda_{r}^{2}$, for states lighter than $T_{R}$ we have $\int_{x_{R}}^{x_{F}} d x x^{4} C_{j, k}^{\text {decay }} \sim m_{t^{\prime}}^{5} m_{j} / \Lambda_{r}^{2}$, and the individual contributions scale linearly with the KK mass, $m_{j}$. It follows that the contribution to the $r^{\prime}$ relic density from KK particle decays grows linearly with the reheat temperature, which sets the effective cutoff for how many KK states give a non-negligible effect.

\subsubsection{Scattering Processes}

We consider now the scattering processes. The contribution to the collision operator from each individual process $g q^{j} \rightarrow r^{\prime} q^{k}$ and for each spin/polarization/color degree of freedom in the initial state takes the form

$$
C_{j, k}^{\text {scatt. }} \equiv \int e^{-\left(E_{q^{j}}+E_{g}\right) / T}\left(4 F \sigma_{g q^{j} \rightarrow r^{\prime} q^{k}}\right) \frac{d^{3} p_{g}}{(2 \pi)^{3} 2 E_{g}} \frac{d^{3} p_{q^{j}}}{(2 \pi)^{3} 2 E_{q^{j}}},
$$

where the scattering cross section is

$$
\sigma_{g q^{j} \rightarrow r^{\prime} q^{k}}=\frac{1}{4 F} \int(2 \pi)^{4} \delta\left(p_{g}+p_{q^{j}}-p_{r^{\prime}}-p_{q^{k}}\right) \overline{\left|\mathcal{M}_{g q^{j} \rightarrow r^{\prime} q^{k}}\right|^{2}} \frac{d^{3} p_{r^{\prime}}}{(2 \pi)^{3} 2 E_{r^{\prime}}} \frac{d^{3} p_{q^{k}}}{(2 \pi)^{3} 2 E_{q^{k}}}
$$

and the flux factor is $F=p_{q^{j}} \cdot p_{g}(=\sqrt{s}|\vec{p}|$, with $|\vec{p}|$ the three-momentum of one of the initial state particles in the $\mathrm{CM}$ ). The relevant diagrams are shown in Fig. 9, and the analytic expression for the cross section is given in Eq. (78) of Appendix B. Following Ref. [43], it is convenient to write $d^{3} p_{g} d^{3} p_{q^{j}}=2 \pi^{2} E_{g} E_{q^{j}} d E_{+} d E_{-} d s$, where $E_{ \pm} \equiv E_{g} \pm E_{q^{j}}$, and $s=m_{j}^{2}+2 E_{g}\left(E_{q^{j}}-p_{q^{j}} \cos \theta\right)$ is the total CM energy squared, while $\theta$ is the angle between the momenta of the initial state particles. It is assumed that $s>\left(m_{r^{\prime}}+m_{k}\right)^{2}$. One finds that the physical region corresponds to $E_{+} \geq \sqrt{s}$, while $\left|E_{-}-E_{+} m_{j}^{2} / s\right| \leq \sqrt{E_{+}^{2}-s}\left(1-m_{j}^{2} / s\right)$. The integrand is independent of $E_{-}$, so that the $E_{-}$integral can be trivially done. The cross section (times the flux factor) depends only on $s$, and the integral over $E_{+}$gives $\int_{\sqrt{s}}^{\infty} d E_{+} \sqrt{E_{+}^{2}-s} e^{-E_{+} / T}=\sqrt{s} T K_{1}(\sqrt{s} / T)$, where $K_{1}$ is a modified Bessel function of the second kind. Thus, we can write

$$
x_{j}^{4} C_{j, k}^{\text {scatt. }}\left[x_{j}\right]=m_{j}^{4} \int_{x_{j}}^{\infty} \frac{8 \pi^{2}}{(2 \pi)^{6}} K_{1}(u)\left(u^{2}-x_{j}^{2}\right)\left(F \sigma_{g q^{j} \rightarrow r^{\prime} q^{k}}\right) d u
$$


where $u \equiv \sqrt{s} / T$ and $x_{j} \equiv m_{j} / T$ are dimensionless variables. The full collision operator associated with scattering of KK fermions is then 6

$$
C^{\text {scatt. }}[x]=\sum_{j, k} \mathcal{N} C_{j, k}^{\text {scatt. }}\left[x_{j}\right]
$$

where $\mathcal{N}=2$ (gluon pol. $) \times\left(N_{c}^{2}-1\right)$ (gluons $) \times 2\left(q^{j}\right.$ spin $) \times N_{c}\left(q^{j}\right.$ color $) \times 2\left(q^{j}\right.$ and $\left.\bar{q}^{j}\right)=$ $8 N_{c}\left(N_{c}^{2}-1\right)$, with $N_{c}=3$. For leptons and weak gauge bosons the multiplicities are different, but as we explained at the beginning of Section 6.1, we neglect such contributions. We consider each SM quark flavor separately in the sum. Also, the allowed processes are of the form $g^{+} q^{-} \rightarrow r^{-} q^{+}$or $g^{+} q^{+} \rightarrow r^{-} q^{-}$, with \pm referring to a KK-parity even/odd state.

In the remainder of this subsection, we provide analytic approximations for the elementary building blocks, $C_{j, k}^{\text {scatt. }}$. The reader mostly interested in the physics results may wish to jump to Section 6.2, where we discuss them.

In the non-relativistic regime $\left(T \lesssim m_{j}\right.$, which implies that the integral in Eq. (26) is dominated by the region $u \sim x_{j}$ ), we have the approximate result

$$
\begin{aligned}
F \sigma_{g q^{j} \rightarrow r^{\prime} q^{k}}^{\text {non } \mathrm{rel}} \approx & \frac{\alpha_{s}}{8 N_{c}} \frac{m_{j}^{2}}{\Lambda_{r}^{2}} \frac{x_{j}^{4}}{\left(u^{2}-x_{j}^{2}\right)^{2}+x_{j}^{2}\left(\Gamma_{j} / T\right)^{2}}\left[(1+\Delta \epsilon) \frac{\tan ^{-1}\left[\frac{\theta\left(\Delta \epsilon, \epsilon_{k, j}\right)}{1+\Delta \epsilon}\right]}{\theta\left(\Delta \epsilon, \epsilon_{k, j}\right)}-1\right] \\
& \times \theta\left(\Delta \epsilon, \epsilon_{k, j}\right)\left\{\left[\left(G_{1 j k}^{R L}\right)^{2}+\left(G_{1 j k}^{L R}\right)^{2}\right](1+\Delta \epsilon)+4 G_{1 j k}^{R L} G_{1 j k}^{L R} \epsilon_{k, j}\right\}
\end{aligned}
$$

where $\theta\left(\Delta \epsilon, \epsilon_{k, j}\right)=\sqrt{(1+\Delta \epsilon)^{2}-4 \epsilon_{k, j}^{2}}, \Delta \epsilon \equiv \epsilon_{k, j}^{2}-\epsilon_{r^{\prime}, j}^{2}, \epsilon_{k, j}=m_{k} / m_{j}$, and $\epsilon_{r^{\prime}, j}=m_{r^{\prime}} / m_{j}$, i.e. we normalize with respect to the incident $t^{j}$ mass. The $G_{i j k}^{R L}$ and $G_{i j k}^{L R}$ are the relevant couplings of the KK-radion (with $i=1$ for $r^{\prime}$ ) to the fermion KK modes [see Eqs. (59), (60) and (54)]. This result is dominated by the s-channel diagram of Fig. 9, and we have explicitly included the $q_{j}$ width, $\Gamma_{j}$, which regulates the limit where the incoming gluon is ultrasoft and the s-channel $q^{j}$ is nearly on shell.

In the relativistic regime (i.e. $T \gg m_{j}$ ) one can instead use

$$
F \sigma_{g q^{j} \rightarrow r^{\prime} q^{k}}^{\mathrm{rel}} \approx \frac{\alpha_{s}}{64 N_{c}} \frac{m_{j}^{2}}{\Lambda_{r}^{2}}\left[\left(G_{1 j k}^{R L}\right)^{2}+\left(G_{1 j k}^{L R}\right)^{2}\right]\left[4 \log \left(\frac{u}{x_{k}}\right)-3\right],
$$

where we took the limit $\sqrt{s} \gg m_{j}$ of the exact cross section given in Eq. 78 of Appendix B, and defined $x_{k} \equiv m_{k} / T$. This result is dominated by the t-channel diagram in Fig. 9. The logarithmic divergence when $m_{k} \rightarrow 0$ is similar to the usual forward scattering singularity associated with the t-channel exchange of a massless particle. In Fig. 10, we show the exact scattering cross section as given in Eq. (78) of Appendix B, together with its nonrelativistic and relativistic approximations, Eqs. (28) and (29), respectively. We choose typical parameters in order to illustrate the precision of the approximations. We will use the

\footnotetext{
${ }^{6}$ Although the couplings of the KK-radion to fermion zero-modes are suppressed by the corresponding fermion mass, its couplings to the KK states are similar for all the light family KK towers. Since the zeromode contribution gives a negligible effect, we can describe the effect of the light families by a multiplicity factor, which would be appropriate in anarchic scenarios.
} 


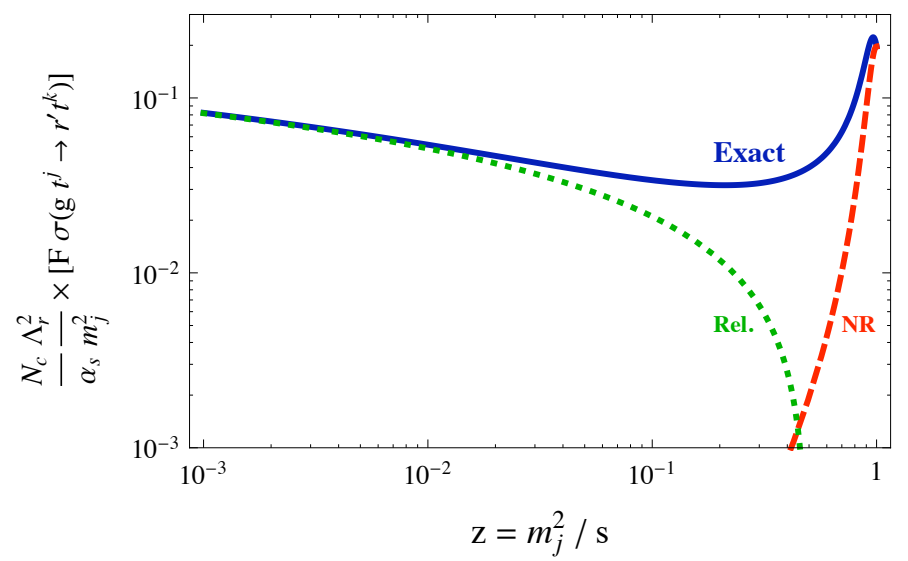

Figure 10: $F \times \sigma\left(g t^{j} \rightarrow r^{\prime} t^{k}\right)$ as a function of $z=m_{j}^{2} / s$, assuming $m_{r^{\prime}} / m_{j}=0.20, m_{k} / m_{j}=$ 0.67, $G_{1 j k}^{R L}=0.50$ and $G_{1 j k}^{L R}=0.41$, which correspond to the decay $g t^{5} \rightarrow r^{\prime} t^{4}$ of the "small warping" benchmark scenario. The solid (blue) curve is the exact result given in Eq. (78) of Appendix B, the dashed (red) curve is the non-relativistic approximation of Eq. (28), and the dotted (green) curve is the relativistic approximation of Eq. (29). We also assumed $\Gamma_{j} / m_{j}=1 / 10$, which is important only for $z \approx 1$.

exact result in our numerical analysis, but the approximate limits provide a more transparent analytic understanding.

For instance, in the non-relativistic limit, the $u$-dependence of the integrand in the collision term of Eq. 26) is given by

$$
g_{\mathrm{NR}} d u=\frac{K_{1}(u)\left(u^{2}-x_{j}^{2}\right)}{\left(\frac{u^{2}}{x_{j}^{2}}-1\right)^{2}+\gamma_{j}^{2}} d u,
$$

where $\gamma_{j} \equiv \Gamma_{j} / m_{j}$. It is instructive to rewrite $g_{\mathrm{NR}}$ as a function of

$$
w=\frac{u^{2}-x_{j}^{2}}{x_{j}^{2} \gamma_{j}}, \quad d u=\frac{x_{j} \gamma_{j}}{2 \sqrt{1+w \gamma_{j}}} d w
$$

so that

$$
g_{\mathrm{NR}} d u=\frac{1}{2} x_{j}^{3} K_{1}\left(x_{j} \sqrt{1+w \gamma_{j}}\right) \frac{w}{w^{2}+1} \frac{d w}{\sqrt{1+w \gamma_{j}}} .
$$

This function determines the support of the scattering collision integral as follows. For $x_{j} \gg 1$ and writing $u=x_{j}+\Delta u$, we see that in this ultra non-relativistic regime $\left(T \ll m_{j}\right)$, we have $K_{1}\left(x_{j} \sqrt{1+w \gamma_{j}}\right)=K_{1}(u) \approx \sqrt{\frac{\pi}{2 x_{j}}} e^{-\left(x_{j}+\Delta u\right)}$, where the use of the asymptotic form of the Bessel function is well justified. The exponential damping $e^{-\Delta u}$ ensures that the support of the integral satisfies $\Delta u \lesssim \mathcal{O}(1)$, and we can truncate the region of integration at $w_{\max } \approx 2 /\left(x_{j} \gamma_{j}\right)$. Thus, using $g_{\mathrm{NR}} d u \approx \frac{1}{2} \sqrt{\frac{\pi}{2}} x_{j}^{5 / 2} e^{-x_{j}} \frac{w d w}{w^{2}+1}$ for $0<w<w_{\max }$ (and 0 otherwise), we can write

$$
x_{j}^{4} C_{j, k}^{\text {non-rel }}\left[x_{j}\right] \approx m_{j}^{4} \frac{2 \pi^{2}}{(2 \pi)^{6}} \sqrt{\frac{\pi}{2}} x_{j}^{5 / 2} e^{-x_{j}} f_{N R}\left(m_{j}, m_{k}, m_{r^{\prime}}\right) \log \left[1+\frac{4}{x_{j}^{2} \gamma_{j}^{2}}\right],
$$


where one should recall that $x_{j}=\left(m_{j} / m_{t^{\prime}}\right) x$, and we defined

$$
f_{N R}\left(m_{j}, m_{k}, m_{r^{\prime}}\right)=\left[\frac{x_{j}^{4}}{\left(u^{2}-x_{j}^{2}\right)^{2}+x_{j}^{4} \gamma_{j}^{2}}\right]^{-1} \times F \sigma_{g t^{j} \rightarrow r^{\prime} t^{k}}^{\text {non-rel }},
$$

a dimensionless function of the masses and couplings only (not temperature) that can be read from Eq. (28).

In the relativistic limit, $x_{j} \ll 1$, the $u$-dependence of the integrand in the collision term of Eq. (26) is given by

$$
g_{\mathrm{R}}=K_{1}(u)\left(u^{2}-x_{j}^{2}\right) \times\left[4 \log \left(\frac{u}{x_{k}}\right)-3\right] .
$$

This function has a maximum approximately at $u_{\max } \approx \frac{3}{2}+1 / \log \left[\frac{3}{2 x_{k}}\right]$. We may then replace $u$ by $u_{\max }$ inside the logarithm in $g_{\mathrm{R}}$. Using $\int_{x_{j}}^{\infty} K_{1}(u)\left(u^{2}-x_{j}^{2}\right)=2 x_{j} K_{1}\left(x_{j}\right) \approx 2$ for $x_{j} \ll 1$, we can then write

$$
x_{j}^{4} C_{j, k}^{\mathrm{rel}}\left[x_{j}\right] \approx m_{j}^{4} \frac{16 \pi^{2}}{(2 \pi)^{6}} f_{R}\left(m_{j}, m_{k}, m_{r^{\prime}}\right)\left[4 \log \left(\frac{u_{\max }}{x_{k}}\right)-3\right],
$$

where $x_{j}=\left(m_{j} / m_{t^{\prime}}\right) x$ and $x_{k}=\left(m_{k} / m_{t^{\prime}}\right) x$, while

$$
f_{R}\left(m_{j}, m_{k}, m_{r^{\prime}}\right)=\frac{\alpha_{s}}{64 N_{c}} \frac{m_{j}^{2}}{\Lambda_{r}^{2}}\left[\left(G_{1 j k}^{R L}\right)^{2}+\left(G_{1 j k}^{L R}\right)^{2}\right]
$$

is temperature-independent. Thus, the contribution to $r^{\prime}$ production via scattering of highly relativistic particles to the l.h.s. of Eq. (15) scales like $x \sim 1 / T$ (up to logarithms). Unlike the decay processes, scatterings that occur all the way up to the reheat temperature, $T_{R}$, contribute uniformly in the $x$-variable. These results follow essentially from the phase space for both cases (taking into account the time dilation factor in the decay widths). Eqs. (33) and (36) provide the basic analytic results to understand the production of $r^{\prime}$ via scattering of KK fermions off the plasma.

In the left panel of Fig. 11 we show $x_{j}^{4} C_{j, k}^{\text {scatt. }}\left[x_{j}\right]$ as given in Eq. 26 for the process $g t^{j} \rightarrow r^{\prime} t^{k}$, as a function of $x_{j}=m_{j} / T$, and factoring out the overall parametric dependence $\frac{8 \pi^{2}}{(2 \pi)^{6}} \frac{\alpha_{s}}{N_{c}} \frac{m_{j}^{6}}{\Lambda_{r}^{2}}$ (solid, blue curve). We also show the non-relativistic (dashed, red) and relativistic (dotted, green) approximations given in Eqs. (33) and (36), respectively. We see that the relativistic approximation works well up to $x_{j} \sim \mathcal{O}(1)$, while for larger $x_{j}$ the non-relativistic approximation may be used. In the right panel of Fig. 11] we show the integral of the previous quantity with respect to $x_{j}$ from $x_{j}=m_{j} / T_{R}$ to $x_{j}=m_{j} / T_{F}$ (solid, blue curve). Here $T_{F}$ is the temperature when $t^{\prime}$ freezes-out, and it is assumed that $m_{j} / T_{F} \gg 1$. Note that this integral differs from the integral appearing in Eq. (15) for the relic density by an overall factor of $\epsilon_{t^{\prime}, j}^{5} \equiv\left(m_{t^{\prime}} / m_{j}\right)^{5}$. The dashed, red curve corresponds to an approximation where the simpler relativistic and non-relativistic expressions are used for $x_{j}<x_{j, k}^{\mathrm{tr}}$ and $x_{j} \geq x_{j, k}^{\mathrm{tr}}$, respectively, for a conveniently chosen transition point $x_{j, k}^{\mathrm{tr}}$. We find that $x_{j, k}^{\mathrm{tr}}=3 m_{j} /\left(2 m_{k}\right)$ gives a reasonably good approximation throughout the whole range, except in a region 

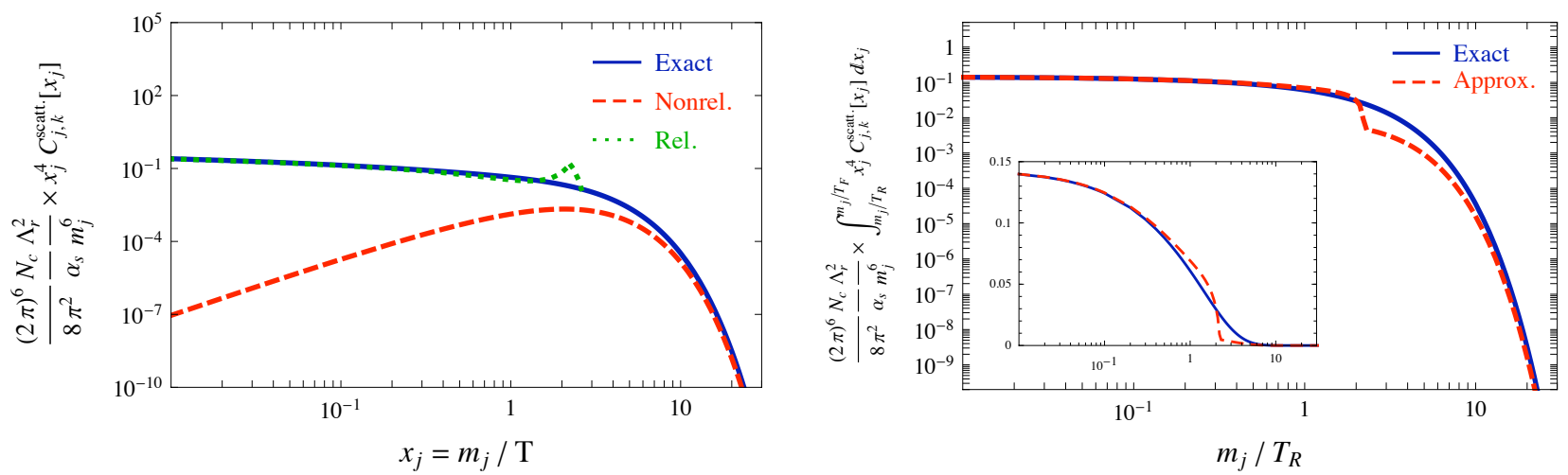

Figure 11: Left panel: Collision operator for the process $g t^{j} \rightarrow r^{\prime} t^{k}$ as a function of $x_{j}=m_{j} / T$. The solid (blue) curve is the exact result with the full cross section, Eq. (26), the dashed (red) curve is the non-relativistic approximation, Eq. (33), and the dotted (green) curve is the relativistic approximation, Eq. (36). Right panel: the integral w.r.t. $x_{j}$ of the previous quantity, as a function of $m_{j} / T_{R}$ (see discussion in the main text). It is assumed that $m_{j} / T_{F} \gg 1$, where $T_{F}$ is the $t^{\prime}$ freeze-out temperature. The solid (blue) curve corresponds to the exact result. The dashed (red) curve is obtained by using the relativistic approximation when $x_{j}<x_{j, k}^{\mathrm{tr}}$ and the non-relativistic approximation when $x_{j} \geq x_{j, k}^{\mathrm{tr}}$, where $x_{j, k}^{\mathrm{tr}}=3 m_{j} /\left(2 m_{k}\right)$. The inset shows the same plot with a linear vertical scale. We have assumed the same parameters as in Fig. 10.

where $m_{j} / T_{R} \sim$ few. In most of this region, however, the contribution to the final $r^{\prime}$ relic density is suppressed since $t^{j}$ becomes non-relativistic. We also see from the rather weak logarithmic dependence on $x_{j}$ of the relativistic expression given in Eq. (36) that, for $m_{j} / T_{R} \ll 1$, the contribution to the integral in the 1.h.s. of Eq. (15) becomes essentially independent of $T_{R}$ [see also inset in the right panel of Fig. 11, and discussion after Eq. (37)]. This is similar to the decay process discussed in Subsection 6.1.1. We can therefore easily compare the contributions form scattering and decays for reheat temperatures that are large compared to the given particle masses. Using the result of Eq. (23) together with Eq. (21) and $\Gamma_{j, k}^{\mathrm{CM}} \sim m_{j}^{3} /\left(16 \pi \Lambda_{r}^{2}\right)$ [see Eqs. 71 or 72$)$, we have

$$
\frac{\int_{x_{R}}^{x_{F}} d x x^{4} C_{j, k}^{\text {decay }}[x]}{\int_{x_{R}}^{x_{F}} d x x^{4} C_{j, k}^{\text {scatt. }}[x]} \sim \frac{\left[4 \pi /(2 \pi)^{3}\right]\left[m_{j}^{6} /\left(16 \pi \Lambda_{r}^{2}\right)\right](3 \pi / 2)}{\left[8 \pi^{2} /(2 \pi)^{6}\right]\left(\alpha_{s} / N_{c}\right)\left(m_{j}^{6} / \Lambda_{r}^{2}\right) \times 10^{-1}} \sim \frac{75 \pi^{2} N_{c}}{20 \alpha_{s}}
$$

where the $10^{-1}$ is read from the right panel of Fig. 11. This estimate indicates that the contribution to $\Omega_{r^{\prime}}$ from the decay processes dominates over the one due to scatterings.

\subsection{Constraints on a Superweakly Interacting KK-Radion}

In the previous subsection we have setup the formalism to compute the production of $r^{\prime}$ in decays of heavy particles, and in scatterings against the plasma. We established that the decay processes dominate over the scattering ones, essentially as a result of phase space considerations, and the fact that at any given energy there are KK states with masses 
of that order, whose decays are important. Thus, we can focus on the production of $r^{\prime}$ via heavy KK mode decays. For a given reheat temperature, $T_{R}$, decays of particles sufficiently heavier than $T_{R}$ give an exponentially small contribution, while decays of particles lighter than $T_{R}$ give a contribution to the integral in the l.h.s. of Eq. (15) of order $(4 \pi) /(2 \pi)^{3} m_{j}^{3} \Gamma_{j, k}^{\mathrm{CM}}\left(m_{t^{\prime}} / m_{j}\right)^{5}(3 \pi / 2)=\left(3 G^{2} / 64 \pi^{2}\right) m_{t^{\prime}}^{5} m_{j} / \Lambda_{r}^{2}$, where $G$ is a dimensionless effective coupling constant for the corresponding decay vertex. The linear dependence on $m_{j}$ translates into a linear dependence on the reheat temperature, which sets the effective cutoff for how many states give a non-negligible contribution to the $r^{\prime}$ relic density. Thus, the contribution from each KK tower is dominated by the states whose masses are around $T_{R}$. In fact, our discussion in Subsection 6.1.1 on the $r^{\prime}$ production due to decays of non-relativistic particles, shows that this contribution can be significant even for masses an order of magnitude (or so) above $T_{R}$. One should also include a multiplicity factor for the internal degrees of freedom for the decaying particles. For instance, for each of the $N_{f}=6$ quark towers, the multiplicity is $2(q$ and $\bar{q}) \times 2$ (spin) $\times N_{c}$ (color). Thus, from the quark towers alone, setting $m_{j} \sim T_{R}$, and using Eq. (15), we can estimate the KK radion density arising from decays of KK modes as

$$
\Omega_{r^{\prime}}^{(1)} h^{2} \sim 0.06 G^{2} \times\left(\frac{10^{16} \mathrm{GeV}}{\Lambda_{r}}\right)^{2}\left(\frac{T_{R}}{10 \mathrm{TeV}}\right)\left(\frac{m_{r}}{300 \mathrm{GeV}}\right)
$$

where we took $g_{*}=86.25$ Note that Eq. (39) is independent of $m_{t^{\prime}}$. A more precise determination is shown in Fig. 12, were we show the region selected by WMAP in the $T_{R}-\Lambda_{r}$ plane, by treating the top towers separately from the light fermion towers (as suggested by the anarchic picture of flavor), and including the decays of KK gauge bosons, as well as the much smaller $r^{\prime}$ production by scatterings. The internal degrees of freedom associated with the top tower give a factor $2 \times 2 \times N_{c}$, while for the light generation towers we have a factor $5 \times 2 \times 2 \times N_{c}+(3+3 / 2) \times 2 \times 2$, which includes the five lighter quarks, three charged leptons and three LH neutrinos. The corresponding factor for KK gauge boson decays is $3\left(N_{c}^{2}-1\right)+3 \times 4$. We also include the $r^{\prime}$ produced in decays of $t^{\prime}$ after its freeze-out, which we previously denoted by $\Omega_{r^{\prime}}^{(2)} h^{2}$, although this contribution is very small (see below).

¿From our discussion in Section 3, one might expect that if the reheat temperature is too large, the correct metric background would correspond to a blackhole solution, and the KK modes, including the radion tower cease to have meaning (in the $4 \mathrm{D}$ dual picture, the theory would be in a deconfined phase). Naively, we expect the critical temperature to be of order $\tilde{k}_{\text {eff }}$, and therefore we show in the left panel of Fig. 12 reheat temperatures up to $\tilde{k}_{\text {eff }} \sim 1 \mathrm{TeV}$. We also show reheat temperatures as low as $T_{R}=15 \mathrm{GeV}$ (which is about the $t^{\prime}$ freeze-out temperature), where the KK-radion density is still small compared to its equilibrium distribution. The radion decay constant that reproduces the observed DM density ranges from $10^{4}-10^{16} \mathrm{GeV}$, depending on $T_{R}$.

However, given that we do not have the corresponding blackhole solution that would allow us to compute the critical temperature, we also show in the right panel of Fig. 12 the result of assuming that the KK theory is the correct description up to temperatures of order its $4 \mathrm{D}$ cutoff (which we estimate to be of order 5-10 TeV). Even in this case, WMAP selects a $\Lambda_{r}$ not much larger than $10^{16} \mathrm{GeV}$. For reheat temperatures above a $\mathrm{TeV}$, lower values of $\Lambda_{r}$ would lead to overproduction of $r^{\prime}$, while larger values would make the $r^{\prime}$ species a 

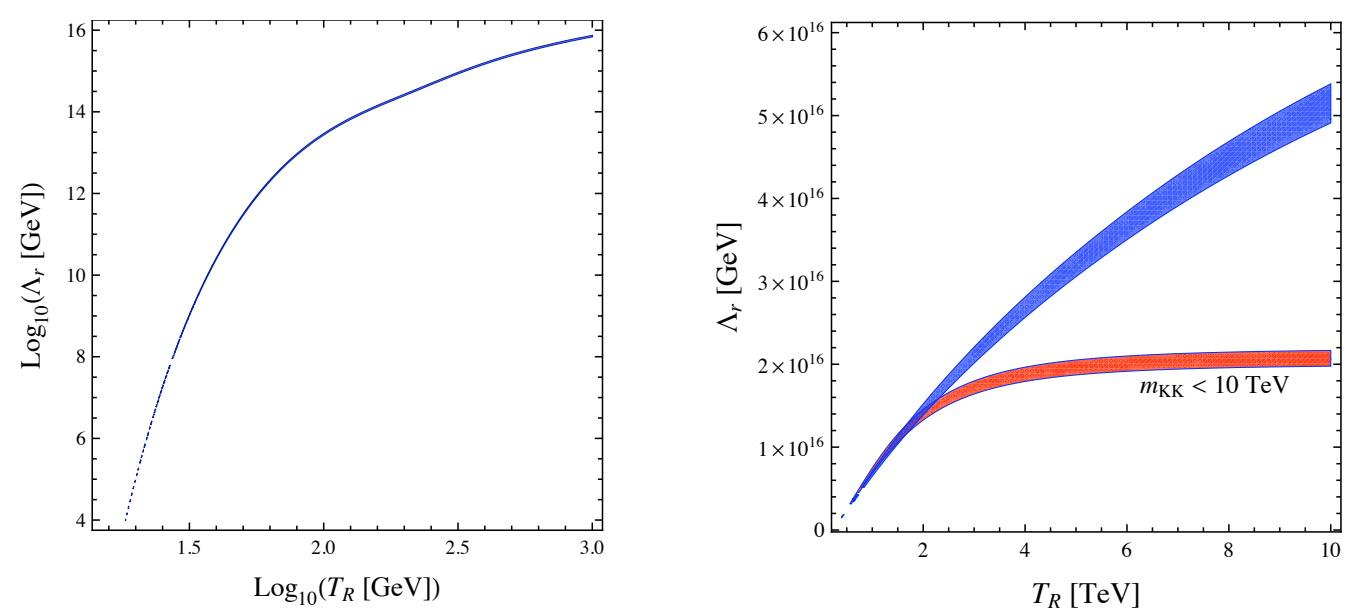

Figure 12: The WMAP constraint in the $\Lambda_{R}-T_{R}$ plane. The left panel shows the allowed region on a logarithmic scale for $15 \mathrm{GeV}<T_{R}<\tilde{k}_{\text {eff }} \sim 1 \mathrm{TeV}$ and $10^{4} \mathrm{GeV}<\Lambda_{r}<10^{16} \mathrm{GeV}$. In the right panel we show, on a linear scale, the WMAP allowed region up to $T_{R}=10 \mathrm{TeV}$ (the cutoff of the KK theory), and illustrate the effect of the number of KK modes included. The blue (upper) region includes contributions from KK states up to an order of magnitude above the maximum reheat temperature shown. The red (lower) region shows the result of truncating the KK tower at $10 \mathrm{TeV}$.

subdominant component of the observed DM density. However, note that, within the model, $\Lambda_{r}$ cannot be arbitrarily large, but is bounded from above by the Planck mass. 7 Thus, quite aside from the likely difficulty in nucleating to the true vacuum from a "deconfined" one if the temperature is above the critical temperature, this result suggests that $T_{R}$ cannot be much higher than the cutoff of the effective theory, or else the KK-radions would be overproduced.

As mentioned above, states somewhat heavier than $T_{R}$ in general cannot be neglected. This raises the question of how to treat these states when $T_{R}$ is close to the cutoff of the effective theory, even assuming that the theory remains in the "confined phase", so that a KK radion can be properly identified. Nevertheless, even if the relevant degrees of freedom above the cutoff are not accurately described by the KK theory, one might expect that their couplings to the KK-radion are still suppressed by $\Lambda_{r}$. One can therefore get an idea by including the contributions from KK states up to about one order of magnitude above the cutoff. The result corresponds to the upper, blue region in the right panel of Fig. 12 , which exhibits the linear dependence on $T_{R}$ previously discussed. However, we also show the result of truncating the KK tower at a cutoff of $10 \mathrm{TeV}$ (lower, red region), as might be appropriate if for some unknown reason the relevant degrees of freedom above that scale are significantly more weakly coupled to the KK-radion than expected from the KK picture. The comparison between the two curves gives an idea of the effect of the heavy states at larger reheat temperatures.

\footnotetext{
${ }^{7}$ Recall that these scales are only effective scales, characterizing the strength of radion and graviton interactions. In the present "small warping" scenario, the physical cutoff of the theory on the UV brane is in fact significantly smaller, of order the 5D Planck mass, $M_{5}$.
} 

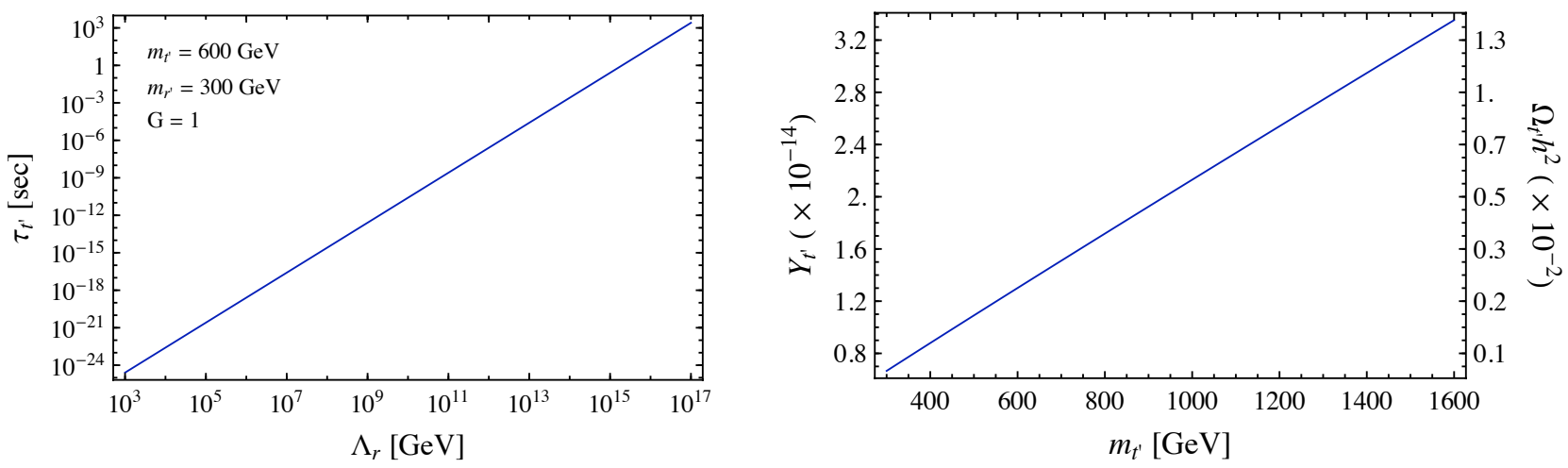

Figure 13: Left panel: $t^{\prime}$ lifetime as a function of $\Lambda_{r}$ for $m_{t^{\prime}}=600 \mathrm{GeV}, m_{r^{\prime}}=300 \mathrm{GeV}$ and $G=1$ (see text). Right panel: $t^{\prime}$ yield, $Y_{t^{\prime}}\left(x_{F}\right)$, as a function of $m_{t^{\prime}}$. For reference, in the right axis we show $\Omega_{t^{\prime}} h^{2} \equiv\left(m_{t^{\prime}} / m_{r^{\prime}}\right) \Omega_{r^{\prime}}^{(2)} h^{2}$ corresponding to the yield shown in the left axis and the mass $m_{t^{\prime}}$ given by the curve.

In this scenario, the large value of $\Lambda_{r^{\prime}}$ would make the NLKP long-lived. For instance, if the NLKP is $t^{\prime}$, and assuming $m_{t^{\prime}}>m_{r^{\prime}}+m_{t}$, it decays via $t^{\prime} \rightarrow r^{\prime} t$ with a decay width given by

$$
\Gamma_{t^{\prime}}=\frac{G^{2} m_{t^{\prime}}^{3}}{16 \pi \Lambda_{r}^{2}} \sqrt{\left[1-\left(\frac{m_{t}}{m_{t^{\prime}}}+\frac{m_{r^{\prime}}}{m_{t^{\prime}}}\right)^{2}\right]\left[1-\left(\frac{m_{t}}{m_{t^{\prime}}}-\frac{m_{r^{\prime}}}{m_{t^{\prime}}}\right)^{2}\right]},
$$

where $G$ is an effective coupling constant [see Eq. (71)]. In Eqs. (76) and (77), we give the decay widths for the KK-parity odd Higgses in the case that one of these is the NLKP. The constraints are similar to the case of a $t^{\prime}$ NLKP, and in the following we concentrate on the latter case. In the left panel of Fig. 13, we show the $t^{\prime}$ lifetime as a function of $\Lambda_{r}$ for $m_{t^{\prime}}=600 \mathrm{GeV}$, and taking $G=1$. The result for other values can be roughly obtained by simple rescaling, according to the prefactor in Eq. (40). These late time decays are potentially dangerous. When massive particles decay into high energy quarks or gluons, the latter rapidly fragment into hadrons, and if the decays of the mother particle occurs at times $\left.10^{-2} \mathrm{~s} \lesssim t \lesssim 10^{4} \mathrm{~s}\right]^{8}$ the scattering off background protons and neutrons via the strong interactions can lead to a dangerous interconversion of background protons and neutrons even after the freeze-out time of the neutron-proton ratio. The effect tends to increase $n / p$, thus increasing the ${ }^{4} \mathrm{He}$ abundance with respect to the standard predictions of BBN. For lifetimes smaller than about $10^{4} \mathrm{~s}$, EM energy deposition is weakly constrained [44], hence the left panel of Fig. 13 suggests that the constraints arise primarily from the hadronic energy injection.

In the right panel of Fig. 13 we show the $t^{\prime}$ yield, $Y_{t^{\prime}}\left(x_{F}\right) \equiv n_{t^{\prime}}\left(x_{F}\right) / s\left(x_{F}\right)$, which is set by the QCD processes of Eq. (18), and depends only on $m_{t^{\prime}}$. In the right axis of the same figure we show the associated $\Omega_{t^{\prime}} h^{2}$, which leads to $\Omega_{r^{\prime}}^{(2)} h^{2}=\left(m_{r^{\prime}} / m_{t^{\prime}}\right) \Omega_{t^{\prime}} h^{2}$, according to

\footnotetext{
${ }^{8}$ At earlier times, such hadrons predominantly scatter off the background photons and electrons, transferring most of their kinetic energy and reaching kinetic equilibrium.
} 
Eq. (17). We can see that the latter one is typically of order $10^{-2}$ or less, and therefore much smaller than the total $\Omega_{r^{\prime}} h^{2}$. This suppression in the $t^{\prime}$ yield allows the picture to be roughly consistent with BBN constraints even when the $t^{\prime}$ decay during or somewhat after BBN. The yield can be constrained as a function of the lifetime of the long-lived relic and its mass, which determine the amount of injected energy and when this injection occurs [45, 46]. We can therefore bound $\Lambda_{r}$ as a function of $m_{t^{\prime}}$ as follows. For a given $m_{t^{\prime}}$ the right panel of Fig. 13 gives the yield, $Y_{t^{\prime}}$. From the results of Ref. [45] we determine how late the relic can decay without being in conflict with BBN constraints (typically around $200 \mathrm{~s}$ ). This can then place an upper bound on $\Lambda_{r}$ via the $t^{\prime}$ decay width. Assuming for illustration that $G=1$ and $m_{r^{\prime}}=300 \mathrm{GeV}$ in Eq. (40), we obtain in this way

\begin{tabular}{|c|c|c|c|c|}
\hline$m_{t^{\prime}}[\mathrm{GeV}]$ & 500 & 800 & 1000 & 1200 \\
\hline$\Lambda_{r}[\mathrm{GeV}] \lesssim$ & $2 \times 10^{16}$ & $5 \times 10^{16}$ & $7 \times 10^{16}$ & $10^{17}$ \\
\hline
\end{tabular}

which gives an upper bound on $\Lambda_{r}$ for a few typical values of $m_{t^{\prime}}$ (or, equivalently, a lower bound on $m_{t^{\prime}}$ for several values of $\Lambda_{r}$ ). Of course, if $m_{t^{\prime}}<m_{r^{\prime}}+m_{t}$, so that we have a three-body decay, the BBN constraint becomes more stringent.

There is a stronger constraint from the late decays of the radions (produced in decays of KK states, analogously to the production of the KK-radions described above). In fact, since the masses and wavefunctions of the radion and KK-radion are nearly identical, their couplings to KK modes are very similar (with appropriate replacements of the semi-degenerate even and odd modes, as dictated by KK parity). We have checked that the number density of radions is very similar to that of KK-radions. The radion yield can then be expressed as

$$
Y_{r} \approx \frac{\Omega_{r^{\prime}} \rho_{c}}{s_{0} m_{r^{\prime}}}
$$

where $\rho_{c}$ and $s_{0}$ are the critical density and entropy of the universe today, respectively, and $\Omega_{r^{\prime}}$ is the DM relic density. For instance, for $m_{r^{\prime}}=500 \mathrm{GeV}$, and assuming $\Omega_{r^{\prime}} h^{2}=0.1$, we get a radion yield of $Y_{r} \approx 7 \times 10^{-13}$. On the other hand, the decays of the radion into $W^{ \pm}$, $Z$, and $h_{+}$give a radion lifetime of

$$
\tau_{r} \approx\left[\frac{m_{r}^{3}}{8 \pi \Lambda_{r}^{2}}\right]^{-1} \approx 0.1 \times\left(\frac{\Lambda_{r}}{10^{15} \mathrm{GeV}}\right)^{2}\left(\frac{500 \mathrm{GeV}}{m_{r^{\prime}}}\right)^{3} \mathrm{sec}
$$

Here we assumed that $m_{W, Z}, m_{h} \ll m_{r}$ and neglected the smaller width into $t \bar{t}$ and massless gauge bosons (we give the exact formulas in Eqs. (84)-(87) of Appendix B.7). For the above reference parameters $\left(m_{r} \approx m_{r^{\prime}}=500 \mathrm{GeV}\right.$ with $\left.Y_{r} \approx 7 \times 10^{-13}\right)$, Ref. [45] gives an upper bound on the radion lifetime of about $0.5 \mathrm{sec}$. Referring to the left panel in Fig. 12, we infer that the reheat temperature should be at most in the few hundred GeV range, but that under this assumption it is possible for KK-radions to account for the DM relic density, while being consistent with BBN constraints (from both radion and $t^{\prime}$ decays ${ }^{9}$ ). Recall

\footnotetext{
${ }^{9}$ Heavier KK radions are more weakly coupled and therefore decay even later. However, they are also produced in smaller quantities, and given that the reheat temperature is significantly below their mass, we expect their number density to be exponentially suppressed.
} 
that a maximum reheat temperature of order a $\mathrm{TeV}$ may be required in order to not exceed the critical temperature for a deconfinement/confinement phase transition, as discussed in Section 3 ,

\section{$7 \quad$ Direct and Indirect Detection}

We now turn to the feasibility of KK-radion detection in either direct or indirect DM searches, as well as collider experiments.

\section{Direct detection:}

As has been mentioned before, most of the interactions between the KK-radion and the SM particles are non-renormalizable, and suppressed by $\Lambda_{r}$. After EWSB, renormalizable interactions with the SM can be induced by terms of the form $\frac{1}{2}[\delta(y-L)+\delta(y+L)] \sqrt{g_{\text {ind }}} \xi \mathcal{R}_{4} H^{\dagger} H$ (subject to the KK-parity symmetry). For simplicity, we restrict here to an exactly IR localized Higgs field, so that the previous operators are IR localized and involve the Ricci scalar constructed from the induced metric. These induce kinetic mixing between the radion and the SM-like Higgs [34, 35]. In the present context they also induce kinetic mixing between the KK-radion and the KK-parity odd (CP-even) $h_{-}$that resides in the inert Higgs doublet, as already mentioned at the end of Section 4. Canonical normalization is achieved by the field redefinitions $r_{ \pm}=\bar{r}_{ \pm} / Z$ and $h_{ \pm}=\bar{h}_{ \pm}+(\delta / Z) \bar{r}_{ \pm}$, where $\delta=6 \sqrt{2} \xi v / \Lambda_{r}$ and $Z^{2}=1+12 \xi(1-6 \xi) v / \Lambda_{r}$, with $v=174 \mathrm{GeV}$. In the following, we will use the notation $r_{+}=r$ and $r_{-}=r^{\prime}$ to denote the radion and $\mathrm{KK}$ radion states. After the previous field redefinitions, the mass matrix becomes non-diagonal. The mass eigenbasis is obtained by the orthogonal transformations:

$$
\left(\begin{array}{c}
r_{ \pm} \\
h_{ \pm}
\end{array}\right)=\left(\begin{array}{cc}
U_{r_{ \pm}, L} & U_{r_{ \pm}, H} \\
U_{h_{ \pm}, L} & U_{h_{ \pm}, H}
\end{array}\right)\left(\begin{array}{c}
\phi_{ \pm, L} \\
\phi_{ \pm, H}
\end{array}\right),
$$

where the subscript $L(H)$ refers to the lighter (heavier) mass eigenstate within the KKparity even and odd sectors, which we denote with the + and - subscripts, as usual. The matrix elements above are explicitly given by

$$
\begin{array}{rlrl}
U_{r_{ \pm}, L} & =\frac{a_{ \pm}-c_{ \pm}-\Delta_{ \pm}}{\sqrt{\left(a_{ \pm}-c_{ \pm}-\Delta_{ \pm}\right)^{2}+4 b_{ \pm}^{2}}}, & U_{r_{ \pm}, H} & =\frac{a_{ \pm}-c_{ \pm}+\Delta_{ \pm}}{\sqrt{\left(a_{ \pm}-c_{ \pm}+\Delta_{ \pm}\right)^{2}+4 b_{ \pm}^{2}}}, \\
U_{h_{ \pm}, L}=\frac{2 b_{ \pm}}{\sqrt{\left(a_{ \pm}-c_{ \pm}-\Delta_{ \pm}\right)^{2}+4 b_{ \pm}^{2}}}, & U_{h_{ \pm}, H}=\frac{2 b_{ \pm}}{\sqrt{\left(a_{ \pm}-c_{ \pm}+\Delta_{ \pm}\right)^{2}+4 b_{ \pm}^{2}}},
\end{array}
$$

where $\Delta_{ \pm}=\sqrt{\left(a_{ \pm}-c_{ \pm}\right)^{2}+4 b_{ \pm}^{2}}$, and 10

$$
a_{ \pm}=\frac{1}{Z^{2}}\left(m_{r_{ \pm}}^{2}+\delta^{2} m_{h_{ \pm}}^{2}\right), \quad b_{ \pm}=\frac{\delta}{Z} m_{h_{ \pm}}^{2}, \quad c_{ \pm}=m_{h_{ \pm}}^{2} .
$$

Here $m_{r_{ \pm}}^{2}$ and $m_{h_{ \pm}}^{2}$ are the mass parameters in the $\left(r_{ \pm}, h_{ \pm}\right)$basis. The physical masses in the KK-parity even and odd sectors are given by

$$
m_{ \pm, L}^{2}=\frac{1}{2}\left(a_{ \pm}+c_{ \pm}-\Delta_{ \pm}\right), \quad m_{ \pm, H}^{2}=\frac{1}{2}\left(a_{ \pm}+c_{ \pm}+\Delta_{ \pm}\right) .
$$

\footnotetext{
${ }^{10}$ Note that, when $a_{ \pm}<c_{ \pm},\left(U_{r_{ \pm}, L}, U_{h_{ \pm}, L}\right) \rightarrow(-1,0)$ and $\left(U_{r_{ \pm}, H}, U_{h_{ \pm}, H}\right) \rightarrow(0,1)$ as $b_{ \pm} \rightarrow 0$. When $a_{ \pm}>c_{ \pm}$, one has instead $\left(U_{r_{ \pm}, L}, U_{h_{ \pm}, L}\right) \rightarrow(0,1)$ and $\left(U_{r_{ \pm}, H}, U_{h_{ \pm}, H}\right) \rightarrow(1,0)$ as $b_{ \pm} \rightarrow 0$.
} 
The DM particle is the lightest KK-parity odd state above, $\phi_{\mathrm{DM}} \equiv \phi_{-, L}$, with mass $m_{\mathrm{DM}} \equiv m_{-, L}$. Now we can identify the interactions relevant for scattering of the DM candidate against nuclei. As shown in [18], there are $r_{-} h_{+} h_{-}$and $r_{+} h_{-} h_{-}$terms of the form

$$
\begin{aligned}
-\frac{2}{\Lambda_{r}} r_{-}\left[\partial_{\mu} h_{+} \partial^{\mu} h_{-}-4 m_{h_{+}}^{2} h_{+} h_{-}\right] & \rightarrow \sum_{\alpha=L, H} \frac{1}{\Lambda_{r}} U_{r_{-}, L} U_{h_{+}, \alpha} U_{h_{-}, L}\left(\square \phi_{+, \alpha}+8 m_{h_{+}}^{2} \phi_{+, \alpha}\right) \phi_{\mathrm{DM}}^{2}, \\
-\frac{2}{\Lambda_{r}} r_{+}\left[\partial_{\mu} h_{-} \partial^{\mu} h_{-}-2 m_{h_{-}}^{2} h_{-} h_{-}\right] & \rightarrow \\
- & \sum_{\alpha=L, H} \frac{1}{\Lambda_{r}} U_{r_{+}, \alpha} U_{h_{-}, L}^{2}\left[\square \phi_{+, \alpha}+\left(2 m_{\mathrm{DM}}^{2}-4 m_{h_{-}}^{2}\right) \phi_{+, \alpha}\right] \phi_{\mathrm{DM}}^{2},
\end{aligned}
$$

where, on the r.h.s, we isolated the terms quadratic in $\phi_{\mathrm{DM}}$. To obtain the above expressions we integrated by parts and assumed that $\phi_{\mathrm{DM}}$ is on-shell: $\square \phi_{\mathrm{DM}}=-m_{\mathrm{DM}}^{2} \phi_{\mathrm{DM}}$. There is also a contribution from the Higgs potential

$$
-\frac{3}{\sqrt{2}} \frac{m_{h_{+}}^{2}}{2 v} h_{+} h_{-}^{2} \rightarrow-\frac{3}{\sqrt{2}} \frac{m_{h_{+}}^{2}}{2 v} \sum_{\alpha=L, H} U_{h_{+}, \alpha} U_{h_{-}, L}^{2} \phi_{+, \alpha} \phi_{\mathrm{DM}}^{2}
$$

The exchanged KK-parity even state can be either $\phi_{+, L}$ or $\phi_{+, H}$. The corresponding Feynman rules for the $\phi_{+, L} \phi_{\mathrm{DM}}^{2}$ and $\phi_{+, H} \phi_{\mathrm{DM}}^{2}$ vertices are, respectively, $\left(2 i / \Lambda_{r}\right)\left(\mathcal{Z}_{L, H} q^{2}+\mathcal{M}_{L, H}^{2}\right)$, where $q$ is the 4-momentum of the KK-parity even state, $\mathcal{Z}_{\alpha}=U_{r_{-}, L} U_{h_{+}, \alpha} U_{h_{-}, L}-U_{r_{+}, \alpha} U_{h_{-}, L}^{2}$, and $\mathcal{M}_{\alpha}^{2}=8 m_{h_{+}}^{2} U_{r_{-}, L} U_{h_{+}, \alpha} U_{h_{-}, L}-\left(2 m_{\mathrm{DM}}^{2}-4 m_{h_{-}}^{2}\right) U_{r_{+}, \alpha} U_{h_{-}, L}^{2}-(3 / 2 \sqrt{2})\left(\Lambda_{r} / v\right) m_{h_{+}}^{2} U_{h_{+}, \alpha} U_{h_{-}, L}^{2}$. Identifying also the Higgs (i.e. $h_{+}$) component in $\phi_{+, L}$ and $\phi_{+, H}$, we obtain the DM-nucleon scattering cross-section

$$
\sigma_{\phi_{\mathrm{DM}} N \rightarrow \phi_{\mathrm{DM}} N} \approx \frac{g_{\mathrm{hNN}}^{2}}{\pi \Lambda_{r}^{2}} \frac{m_{N}^{2}}{m_{+, L}^{4} m_{+, H}^{4}\left(m_{N}+m_{\mathrm{DM}}\right)^{2}}\left(U_{h_{+}, L} \mathcal{M}_{L}^{2} m_{+, H}^{2}+U_{h_{+}, H} \mathcal{M}_{H}^{2} m_{+, L}^{2}\right)^{2}
$$

In the above, we neglected the coupling of the radion to the nucleon compared to the Higgsnucleon coupling, $g_{\mathrm{hNN}} \approx 340 \mathrm{MeV} / 246 \mathrm{GeV}$ [47. Note, however, that for $\Lambda_{r} \sim 2 \mathrm{TeV}$, the radion-nucleon coupling, of order $m_{N} / \Lambda_{r}$, need not be completely negligible (it can be easily incorporated if wanted).

In the left panel of Fig. 14, we show contours of constant $\sigma_{\phi_{\mathrm{DM}} N \rightarrow \phi_{\mathrm{DM}} N}$ in the $\xi-m_{r_{-}}$plane, taking $\Lambda_{r}=2 \mathrm{TeV}$ and $m_{h_{-}}=1 \mathrm{TeV}$. Note that, for practical purposes, the DM physical mass is very close to $m_{r^{\prime}} \equiv m_{r_{-}}$for $m_{r^{\prime}} \lesssim m_{h_{-}}$, and very close to $m_{h_{-}}$otherwise. Thus, the horizontal axis in the plot can be readily interpreted in terms of $m_{\mathrm{DM}}$. The dashed (white) lines correspond to constant $\left|U_{r_{-}, L}\right|^{2}$ (the $r_{-}$content of the DM matter). As discussed at the end of Section 4 , the $h_{-}$content, given by $1-\left|U_{r_{-}, L}\right|^{2}$ should be small, or else the DM relic density will be too small due to efficient annihilation via the Higgs couplings. However, we see that there is a region where the DM is mostly $r_{-}$, with a sizable nucleon cross section (e.g. below the dashed line of $\left|U_{r_{-}, L}\right|^{2}=0.9$ ). In fact, the current direct DM searches may have a sensitivity to this region, as shown by the gray region corresponding to the most recent exclusion by the XENON100 experiment [48]. 

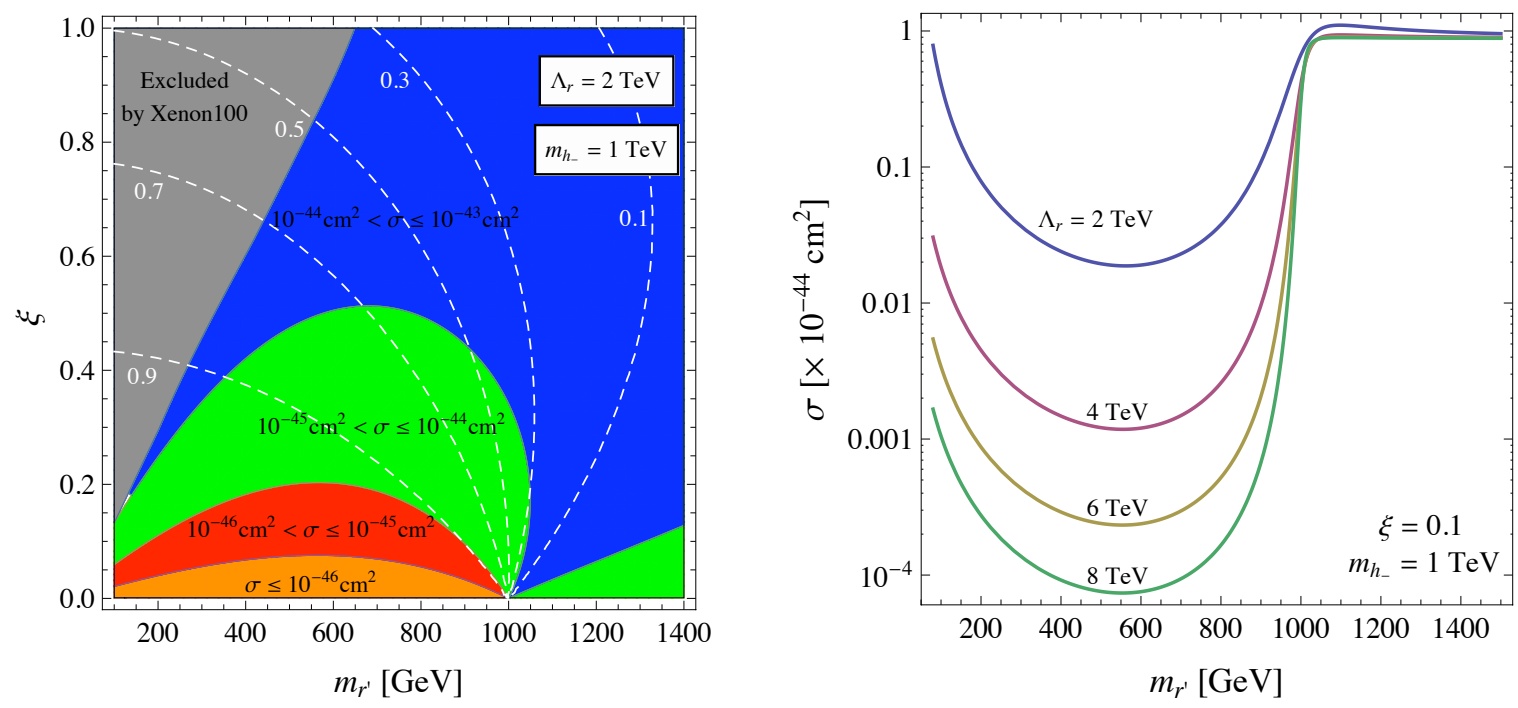

Figure 14: Left panel: Contours of constant $\sigma_{\phi_{\mathrm{DM}} N \rightarrow \phi_{\mathrm{DM}} N}$ in the $\xi-m_{r_{-}}$plane, as given in Eq. (47), for $\Lambda_{r}=2 \mathrm{TeV}$. Also shown is the region currently excluded by XEONO100 (gray). The dashed, white lines represent the $r_{-}$content of the DM candidate, $\left|U_{r_{-}, L}\right|^{2}$ (with $\left|U_{h_{-}, L}\right|^{2}=1-\left|U_{r_{-}, L}\right|^{2}$ being the $h_{-}$content). Right panel: Nucleon cross section as a function of $m_{r_{-}}$, for different values of $\Lambda_{r}$, and for constant $\xi=0.1$. Both figures correspond to $m_{h_{-}}=1 \mathrm{TeV}$.

In the right panel of Fig. 14, we show curves of the nuclear cross section for several values of $\Lambda_{r}$ for fixed $\xi=0.1$ and $m_{h_{-}}=1 \mathrm{TeV}$. The plateau for $m_{r^{\prime}}>m_{h_{-}}$corresponds to the limit in which the DM is pure $h_{-}$, where Eq. 477) reduces to

$$
\sigma_{\phi_{\mathrm{DM}} N \rightarrow \phi_{\mathrm{DM}} N} \approx \frac{9 g_{\mathrm{hNN}}^{2}}{8 \pi v^{2}} \frac{m_{N}^{2}}{\left(m_{N}+m_{\mathrm{DM}}\right)^{2}},
$$

with $m_{\mathrm{DM}}=m_{h_{-}}=1 \mathrm{TeV}$ (we also assumed $\Lambda_{r} \gg v$, so that the exchanged $h_{+}$has no radion admixture).

\section{Indirect detection:}

Regarding indirect detection, the most interesting annihilation channels are those that lead to positrons, neutrinos or photons. The direct annihilation cross section into $e^{+} e^{-}$pairs takes the form $\mathrm{v} \sigma_{r^{\prime} r^{\prime} \rightarrow e^{+} e^{-}} \approx\left(G_{1100}^{2} / 4 \pi\right)\left(m_{e}^{2} / \Lambda_{r}^{4}\right)$, where $G_{1100}$ is the coupling for the contact interaction between two KK-radions and $e^{+} e^{-}$(see second diagram in Fig. 1). In anarchic scenarios, due to EWSB effects and the subsequent mixing with the electron KK resonances, the coupling $G_{1100}$ can be as large as $\mathcal{O}(30)$. However, the $m_{e}^{2}$ factor makes this channel extremely suppressed. Mixing with the Higgs does not help since it also couples to electrons via the small electron Yukawa coupling. Similarly, the direct annihilation rate into neutrinos is negligible. Softer positrons/neutrinos can be produced in the decay products of the dominant $t \bar{t}$ and Higgs (or gauge boson) annihilation channels, but these the fluxes are expected to be small. For instance, given that the spin-independent DM-nucleon cross section is bounded from above by about $10^{-43} \mathrm{~cm}^{2} \approx 10^{-7}$ pb for $m_{\mathrm{DM}} \sim 300 \mathrm{GeV}$, neutrinos from DM annihilations in the Sun would produce at most a couple of neutrino events 

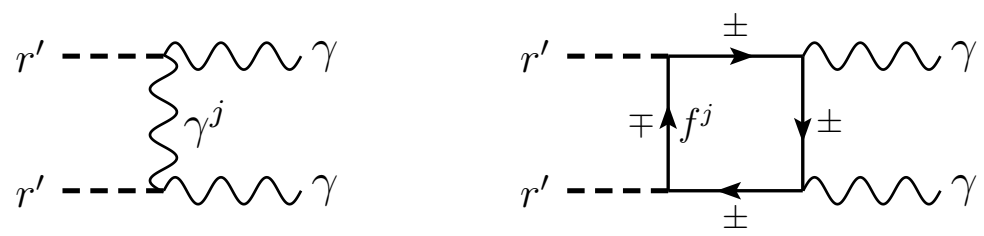

Figure 15: Tree-level (from heavy gauge boson exchange) and 1-loop (from KK fermions) contributions to $r^{\prime} r^{\prime} \rightarrow \gamma \gamma$. The \pm indicate the allowed KK-parity combinations.

per year [49] at neutrino telescopes such as IceCube/Deep Core. This is well below the atmospheric neutrino background, and the detection in this channel is not likely.

We therefore focus on the photon flux from KK-radion annihilations, starting with a possible line signal. There are tree-level direct annihilations from the exchange of KKphotons, as shown in Fig. 15. A given KK-parity odd photon induces the effective dimension8 operator $-2 g_{1 j 0}^{2} /\left(\Lambda_{r}^{2} m_{j}^{2}\right) \partial^{\mu}\left[r^{\prime} F_{\mu \nu}\right] \partial_{\alpha}\left[r^{\prime} F^{\alpha \nu}\right]$, where $g_{1 j 0}$ is the coupling between the KKradion, the KK-photon with mass $m_{j}$, and the massless photon, as given in Ref. [18]. Since both $\Lambda_{r}$ and $m_{j}$ may be expected to be of order a couple $\mathrm{TeV}$, this contribution is small (we give the full cross-section for any $m_{j}$ in Eq. (68) of Appendix B). There are also 1-loop contributions, e.g. from fermion KK loops, as shown in the right diagram of Fig. 15. Imposing only $U(1)_{\text {em }}$ gauge invariance, the effect from each KK level is logarithmically divergent by power counting, which would seem to lead to a linear divergence after summing over KK modes. 11 Nevertheless, as pointed out in Ref. [50] for the case of the linear radion couplings to $\gamma \gamma$ (where a triangle rather than a square diagram is involved), these divergences simply renormalize the 5D operator $\sqrt{g} F_{M N} F^{M N}$. Unfortunately, this operator does not lead to interactions involving two KK-radions and two gauge zero-modes, as pointed out in Ref. [18]. The remaining finite 1-loop pieces contribute to the dimension- 8 operator quoted above, and result in a negligible effect.

Nevertheless, one can still write 5D operators that do lead to the $\left(r^{\prime}\right)^{2} \gamma \gamma$ interaction if the stabilizing scalar field is involved (since the KK-radion is really an admixture of metric and stabilizing scalar fluctuations). However, we find that a more important contribution can arise from an IR localized operator involving instead the extrinsic curvature. For instance, consider the operator $[\delta(y+L)+\delta(y-L)] \sqrt{g_{\text {ind }}}\left(\eta / 8 \Lambda_{5}^{3}\right) K^{2} F_{\mu \nu} F^{\mu \nu}$, where $\Lambda_{5}$ is the $5 \mathrm{D}$ cutoff, the indices are contracted with the induced metric, and $K=4\left[A^{\prime}(y)+\partial_{y} F(x, y)\right]$ is the trace of the extrinsic curvature, including radion fluctuations about the background, as parameterized in Refs. [50, 18]. Reduction to 4D with the flat photon wavefunction, $1 / \sqrt{2 L}$, as well as the KK-radion wavefunction, which satisfies $F_{0}(L) \approx 1$ and $F_{0}^{\prime}(L) \approx 2 A^{\prime}(L)$, leads

\footnotetext{
${ }^{11}$ Although there is a double sum in the square diagram, the "diagonal" couplings, i.e. those for the pairs of KK-parity even and odd fermions with closer masses are largest, while the corresponding couplings rapidly decrease as the KK-fermions become more separated in mass [18].
} 
to the dimension- 6 operator 12

$$
-\frac{e^{2} \kappa}{8 \Lambda_{r}^{2}} r^{\prime 2} F_{\mu \nu} F^{\mu \nu}
$$

where $e^{2} \kappa \approx 64 \eta /\left(k_{\text {eff }} L\right) \times\left(k_{\text {eff }} / \Lambda_{5}\right)^{3}$, with $k_{\text {eff }} \equiv A^{\prime}(L)$. NDA suggests that the unknown dimensionless coefficient, $\eta$, can be as large as $3 \pi / 2$ [36]. Taking also $k_{\text {eff }} L \approx 60$ and $\Lambda_{5} \sim 5 k_{\text {eff }}$, one estimates $e^{2} \kappa \approx 0.04$. Notice that the same operator contains a term without radion insertions, which renormalizes the electric charge. However, this effect is very small and we neglect it in the following.

The interaction of Eq. (49) leads to a direct $\gamma \gamma$ annihilation cross-section given by

$$
\left\langle\sigma_{2 \gamma} v / c\right\rangle \approx\left(\frac{m_{\mathrm{DM}}}{\Lambda_{r}}\right)^{4} \frac{3 \pi \alpha^{2} \kappa^{2}}{m_{\mathrm{DM}}^{2}}
$$

where $m_{\mathrm{DM}}=m_{r^{\prime}}$, and $\alpha=e^{2} / 4 \pi$ is the fine structure constant. In the following, we do not include the additional $\gamma Z$ channel, nor any mixing with the Higgs.

The differential photon flux from the galactic center can be written as

$$
\frac{d \Phi_{\gamma}}{d E}=5.66 \times 10^{-12} \mathrm{~cm}^{-2} \mathrm{~s}^{-1} \frac{d N_{\gamma}}{d E}\left(\frac{\left\langle\sigma_{2 \gamma} v / c\right\rangle}{1 \mathrm{pb}}\right)\left(\frac{1 \mathrm{TeV}}{m_{\mathrm{DM}}}\right)^{2} \bar{J}(\Delta \Omega) \Delta \Omega,
$$

where $d N_{\gamma} / d E=2 \times \delta\left(E-m_{\mathrm{DM}}\right)$ is the differential photon yield in the $2 \gamma$ annihilation channel, $\bar{J}(\Delta \Omega) \equiv(1 / \Delta \Omega) \int_{\Delta \Omega} J(\psi) d \Omega$ integrates over the angular acceptance of the detector $\Delta \Omega$, and $J(\psi)$ is conventionally defined as

$$
J(\psi)=\frac{1}{8.5 \mathrm{kpc}}\left(\frac{1}{0.3 \mathrm{GeV} / \mathrm{cm}^{3}}\right)^{2} \int_{0}^{\infty} d l \rho^{2}(r),
$$

where $r^{2}=l^{2}+r_{0}^{2}-2 l r_{0} \cos \psi$, with $r_{0} \approx 8.5 \mathrm{kpc}$ the distance from the Earth to the galactic center. The integration is along the line of sight, $d l$, and encodes the information about the DM distribution, assuming a spherical DM halo of energy density $\rho(r)$. The quantity $\bar{J}(\Delta \Omega)$ can vary over several orders of magnitude depending on the halo model. We will use here the Navarro-Frenk-White (NFW) profile [51], but the results for other profiles can be obtained by simple rescaling. ${ }^{13}$ We have also used the standard DM density at the Sun's location of $0.3 \mathrm{GeV} / \mathrm{cm}^{3}$, although it has been recently argued that it should be somewhat larger [53. We take $\Delta \Omega=10^{-5} \mathrm{sr}$, as would be relevant for the HESS and Fermi-LAT $\gamma$-ray experiments. We then have $\bar{J}(\Delta \Omega) \Delta \Omega \approx 0.15$.

We take into account the detector energy resolution by convoluting

$$
\left.\frac{d \Phi_{\gamma}}{d E_{0}}\right|_{\text {measured }}=\int d E G\left(E, E_{0}\right) \frac{d \Phi_{\gamma}}{d E},
$$

\footnotetext{
${ }^{12}$ We point out that, in spite of being localized on the IR boundaries, the above operator leads to a non-vanishing linear coupling between the radion field and two photons: $\int d^{5} x \sqrt{g} F(x, y) T \rightarrow$ $-\int d^{4} x\left(8 \eta k_{\text {eff }}^{2} / L \Lambda_{5}^{3}\right)\left(r / \Lambda_{r}\right) F_{\mu \nu} F^{\mu \nu}$, where $T$ is the trace of the stress-energy tensor induced by the operator. This result holds even if the gauge fields are strictly IR localized, and therefore can be interpreted as an explicit breaking of conformal invariance in a dual $4 \mathrm{D}$ picture.

${ }^{13}$ It has been claimed that cored profiles are the rule [52, in which case the photon flux would be significantly smaller.
} 


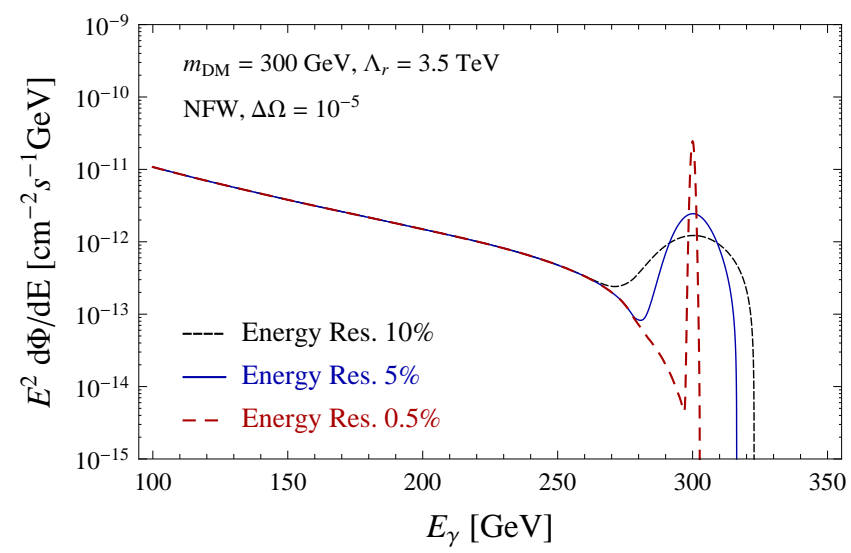

Figure 16: Continuum photon flux and $2 \gamma$-line signal from KK-radion annihilation at the center of the galaxy. For the line signal, we show three detector energy resolutions: $10 \%$ (short dashed, black line), $5 \%$ (solid, blue line) and $0.5 \%$ (dashed, red line).

where, following [54], we take a Gaussian kernel, $G\left(E, E_{0}\right)=1 /\left(\sqrt{2 \pi} E_{0} \sigma\right) e^{-\left(E-E_{0}\right)^{2} /\left(2 \sigma^{2} E_{0}^{2}\right)}$ with $\sigma=\xi / 2.3$, and $\xi$ is the detector's relative energy resolution. We show the line signal in Fig. 16 for $m_{\mathrm{DM}}=300 \mathrm{GeV}$ and $\Lambda_{r}=3.5 \mathrm{TeV}$ (the rest of the parameters are those for the "strong warping" benchmark scenario). We also show in the figure the continuum $\gamma$-ray flux spectrum as computed by micrOMEGAs 2.4 [55], which also reproduces our result for the DM relic density (and, for the previous parameters, gives $\Omega_{r^{\prime}} h^{2} \approx 0.11$ ). We see that the line feature is distinguishable from the continuum spectrum signal, perhaps even for the current typical detector energy resolutions of about $10 \%$.

It is interesting to compare to UED scenarios [56]. The continuum photon signal was computed in Ref. [57], where it was pointed out that the resulting spectrum is rather flat up to the DM mass. This is due to the large branching fraction for direct annihilation of the $\mathrm{KK} B^{(1)}$ into lepton pairs, which results in a hard photon spectrum. In our case, with annihilations mostly into top pairs, gauge bosons and the Higgs, the resulting spectrum is softer than in the UED scenario. The photon line signal, which arises from a calculable 1-loop effect, was also studied in UEDs [58, 54]. It was found that the line peak could be easily seen provided the detector energy resolution was small enough. In the KK-radion scenario, we point out that an incalculable 5D interaction can also lead to a prominent line signal. One should also remark that the KK-radion can be lighter than $B^{(1)}$, which can be very interesting from the point of view of FERMI-LAT, which has sensitivity for photon energies between about 20 and $300 \mathrm{GeV}$. We find that the integrated photon flux from the KK-radion signal, in the above range, is $\Phi_{\gamma} \approx 3 \times 10^{-12} \mathrm{~cm}^{-2} \mathrm{~s}^{-2}$, which is somewhat more than an order of magnitude smaller than FERMI-LAT's sensitivity of about $10^{-10} \mathrm{~cm}^{-2} \mathrm{~s}^{-2}$. However, one should recall that there are large uncertainties associated with the DM halo model profile that can change the signal by orders of magnitude.

Collider signals:

Finally, we comment briefly on the collider signals of our setup. Regarding the more generic KK phenomenology, which can be expected to share features of the widely studied RS scenarios (see [59] for a review), we only note that the generic 1st KK-level states (which 
are KK-parity odd) and 2nd KK level states (which are KK-parity even) can lead to signals both with and without missing energy. In this respect, the situation is rather different from supersymmetric scenarios with R-parity. In addition, we have emphasized that KK-parity even and odd states come in relatively degenerate pairs, which allows to access both types of states almost simultaneously. This can be contrasted to the UED case with KK-Parity: although the first KK-level UED particles always lead to missing energy signals [60], and the second level KK states can lead to pure SM particle final states [60, 61], the latter are about a factor of 2 heavier than the former in 5D constructions [2] (in 6D constructions such as the "chiral square" of Ref. [4] this factor is just $\sqrt{2}$, which can be further diluted due to radiative effects [62]). Note also that there are significantly lighter KK-parity odd fields (discussed next) that mitigate the requirement of pair-production, so that the reach in KK-parity even and odd states should be similar.

Our main focus here is on the DM-related collider signals. Of particular importance are the KK-parity odd states such as the first excited top resonance (probably the $S U(2)$ singlet), or perhaps the "inert" Higgs doublet. These states may be expected to be parametrically lighter than the remaining 1st KK-level states, and thus provide the most promising portal for $r^{\prime}$ production. For instance, in the "strong benchmark scenario" discussed in previous sections, we have $m_{r^{\prime}} \sim 300 \mathrm{GeV}$ and $m_{t^{\prime}} \sim 600 \mathrm{GeV}$. The vector-like quarks have a strong production cross section at the LHC of a couple picobarn [63, 64]. Their main decay is $t^{\prime} \rightarrow t r^{\prime}$, thus leading to a $t \bar{t}+\mathbb{E}_{T}$ signal. This topology was studied in a model-independent way in [65]. Given the large $\mathbb{E}_{T}$, which can be used for tagging, one can consider the case where both top quarks decay hadronically, thus allowing for their full reconstruction. It was shown in the previous study that a judicious set of cuts and kinematic variables can allow for a $t^{\prime}$ discovery at the LHC with less than $10 \mathrm{fb}^{-1}$, and in fact that both $m_{t^{\prime}}$ and $m_{r^{\prime}}$ can be statistically measured. Interestingly, within the context of our model, we can have access to the LKP mass by studying the closely related radion mode, that behaves in many ways like a Higgs. For $m_{r} \approx m_{r^{\prime}} \sim 300 \mathrm{GeV}$, the radion will be produced by gluon fusion and has a significant branching fraction into $Z Z$ [34, 35, 66], thus allowing for a clean mass measurement in the fully charged $4 l$ channel. We can therefore get an interesting hint for the expected degeneracy between $r$ and $r^{\prime}$, and the relatively precise knowledge of the radion mass can be used to study in more detail the properties of the missing energy signal.

It would also be extremely interesting to measure the radion decay constant, which sets the total width for both $t^{\prime}$ and the radion. For the above masses, and for $\Lambda_{r} \sim 3-4 \mathrm{TeV}$, as suggested by the relic density scenario discussed in Section 4, these widths are of order a fraction of a GeV. This may be too small to be measured at a hadron collider, but would be accessible in a lepton machine. Nevertheless, one might be able to infer $\Lambda_{r}$ from a measurement of the radion production cross section. This could be a first test of the required properties to account for the DM relic density. Eventually, we expect that a compelling case for the identity of DM could be made based on collider measurements.

In previous sections, we also considered scenarios with radion decay constants much larger than the $\mathrm{TeV}$ scale. In the coannihilation scenario of Section 5 , with $\Lambda_{r}$ as large a $10^{4} \mathrm{TeV}$, the $t^{\prime}$ still decays promptly but the produced tops (and even the W's) are expected to be off-shell. The analysis of this signal is expected to be challenging. It may also be possible for the $t^{\prime}$ to decay, via intergenerational mixing, into the lighter families. On the other hand, the non-thermal scenario studied in Section 6 can potentially lead to a $t^{\prime}$ that is long-lived 
on collider time scales, if $\Lambda_{r} \gtrsim 10^{10} \mathrm{GeV}$. The same applies to the "inert" Higgs doublet (which has a charged component) if it turns out to be the NLKP. These stable, charged states would lead to spectacular signals at the LHC.

\section{Conclusions}

In this work we have considered warped extra-dimensional scenarios with a KK-parity symmetry that makes the lightest KK-parity odd particle stable and hence a DM candidate. The LKP is expected to be the first KK excitation of the radion field, which is assumed to be stabilized at tree-level by the Goldberger-Wise mechanism. The radion mass is expected to be of order a few hundred $\mathrm{GeV}$, but its interactions -which are controlled by the radion decay constant $\Lambda_{r}$ - can vary over orders of magnitude. We considered a number of scenarios.

\section{Thermal KK dark matter}

1. The desired KK-radion relic abundance can naturally arise when $\Lambda_{r}$ is in the multi-TeV range. EWSB plays an important role here in that it induces strong mixing in the top KK tower, and as a result a relatively large effective coupling between the KK-radions and a $t \bar{t}$ pair that partially offsets the suppression from $\Lambda_{r}$.

2. The presence of light fermion resonances (e.g. the first KK excitation of the RH top) can, via coannihilation effects, deplete the number density of thermal KK radions down to acceptable levels for even larger radion decay constants of order $10^{4} \mathrm{GeV}$. Provided the required degree of degeneracy is present (of order 10-15\%), the relevant annihilation cross section may be purely controlled by the QCD interactions.

3. Alternatively, a small KK-parity odd Higgs component of the DM candidate, can be rather efficient in enhancing the DM self-annihilation cross section. This is due to the relatively strong interactions of the CP-even (but KK-parity odd) KK Higgs with the fermion KK states. If this Higgs component is sizable, one expects that these KK particles would constitute only a fraction of the observed DM density. However, it is possible that a combination of radion-Higgs mixing and a large radion decay constant can result in the required annihilation cross-section to obtain the DM relic abundance via the freeze-out paradigm.

\section{Non-thermal KK dark matter}

4. In UED-like scenarios, where the bulk curvature is small compared to the 5D Planck mass, the radion decay constant can be as large as the Planck scale. If the KKradion interactions are so weak that the KK-radions never reach thermal equilibrium, a lower bound on the radion decay constant can be set from the requirement that these non-thermal KK radions do not overclose the universe, for a given reheat temperature. In such a case, there is a population of long-lived radions and NLKPs that can decay around the time of Big Bang nucleosynthesis. Consistency with BBN puts an upper bound on $\Lambda_{r}$, which controls their lifetimes. This constraints implies 
$\Lambda_{r} \lesssim \mathcal{O}\left(10^{15} \mathrm{GeV}\right)$, while the reheat temperature should be around the EW scale or somewhat below. We also noted that there may be another upper bound on the reheat temperature, of order $\mathrm{TeV}$, to avoid a dangerous high-temperature deconfinement/confinement phase transition. The point is that in the scenario with very large radion decay constants, the rate of bubble nucleation of low-temperature vacua would be extremely suppressed, and furthermore the associated reheat temperature after the transition may not be high enough to allow for a successful BBN. These constraints can be avoided if the universe never reheated above the critical temperature, which can nevertheless be high enough to allow the non-thermal DM KK-radion picture above to be realized.

We also explored the prospects for direct and indirect detection of KK-radion DM. If the scenario has a relatively low radion decay constant, direct detection is feasible and a region of parameter space is already excluded by XENON100. A small KK-Higgs component is essential. For indirect detection experiments, annihilation into positrons or neutrinos is expected to give rather small fluxes (both from direct annihilations, as well as in decay chains of the dominant $t \bar{t}$ /gauge/Higgs channels). However, there may be an interesting photon signal that includes a prominent photon line. This line signal can naturally fall in the energy range of sensitivity of Fermi/LAT, and the total flux may be measurable for cuspy DM halo models.

We also point out that warped scenarios with KK-parity would lead to interesting collider signals, both with and without missing energy. In particular, one may be able to probe the characteristic degeneracy between the DM candidate and the radion mode (which leads to signals similar to the Higgs), at least if the first KK-parity odd excitation of the top is in the expected few hundred $\mathrm{GeV}$ range. The scenarios with very large radion decay constant can lead to highly ionizing charged tracks.

\section{Acknowledgments}

We would like to thank Hsin-Chia Cheng, Hooman Davoudiasl, Piyush Kumar and Jessie Shelton for discussions. A.M. is supported by the US department of Energy under contract DE-FG02-91ER406746. E.P. is supported by DOE grant DE-FG02-92ER40699.

\section{A Radion-fermion interactions and EWSB}

The most general interaction Lagrangian of a single $r_{i}$ and two fermions can be written as

$$
\mathcal{L}_{\mathrm{r} \psi \psi}=-\sum_{i j k=0}^{\infty} \frac{r_{i}}{\Lambda_{r}}\left\{g_{i j k}^{L L} \bar{\psi}_{L}^{j} i \stackrel{\leftrightarrow}{\phi} \psi_{L}^{k}+g_{i j k}^{R R} \bar{\psi}_{R}^{j} i \stackrel{\leftrightarrow}{\not} \psi_{R}^{k}-\left(m_{i j k}^{R L} \bar{\psi}_{R}^{j} \psi_{L}^{k}+\text { h.c. }\right)\right\}
$$

where $\bar{\psi} \overleftrightarrow{\not} \chi \equiv \frac{1}{2}\left[\bar{\psi} \gamma^{\mu} \partial_{\mu} \chi-\left(\partial_{\mu} \bar{\psi}\right) \gamma^{\mu} \chi\right]$, the $g_{i j k}^{L L}$ and $g_{i j k}^{R R}$ are dimensionless, and the $m_{i j k}^{R L}$ have dimensions of mass (these coefficients satisfy the symmetry properties $g_{i j k}^{L L}=g_{i k j}^{L L}, g_{i j k}^{R R}=g_{i k j}^{R R}$ and $m_{i j k}^{L R}=m_{i k j}^{R L}$ ). The sum runs over all fermionic mass eigenstates (as well as the $r_{i}$ ). In 
general, the $\psi^{j}$ are not gauge eigenstates, since the KK towers associated with different bulk fields mix when the Higgs gets a VEV, thus inducing EWSB mixing mass terms of the form

$$
\mathcal{L}_{m}^{\mathrm{EWSB}}=-\sum_{j k=0}^{\infty}\left(\tilde{m}_{j k}^{R L} \bar{\chi}_{1, R}^{j} \chi_{2, L}^{k}+\text { h.c. }\right)
$$

Here $\chi_{1}$ and $\chi_{2}$ stand for two different bulk fields in the gauge eigenbasis (the $S U(2)_{L}$ singlets and doublets of the 5D SM), while the $\tilde{m}_{j k}^{R L}$ are proportional to the Higgs VEV, $v$. For a Higgs localized on the IR brane, these mixing mass terms are given by $\tilde{m}_{j k}^{R L}=$ $v\left(Y_{5 D} / L\right) \frac{1}{2}\left[1+(-1)^{j+k}\right] f_{1, R}^{j}(L) f_{2, L}^{k}(L)$, where $v=174 \mathrm{GeV}, f_{1, R}^{j}$ and $f_{2, L}^{k}$ are the properly normalized fermion wavefunctions evaluated on the IR boundary at $y=L$, and the factor in square brackets reflects the KK parity symmetry of our setup. Perturbatively, the 5D Yukawa coupling, $Y_{5 D}$, is related to the $4 \mathrm{D}$ Yukawa coupling by $Y_{4 D}=\left(Y_{5 D} / L\right) f_{L}^{0}(L) f_{R}^{0}(L)$, where $m_{\psi^{0}}=Y_{4 D} v$ is the corresponding SM fermion mass. However, one should match to the observed fermion mass after diagonalization of the full KK mass matrix. This is especially true in "anarchic" scenarios where the 5D Yukawa couplings are large and mixing with the KK states can be important.

There are linear interactions of the $r_{i}$ that have the same origin as the EWSB mass terms of Eq. (55) and that take the form

$$
\mathcal{L}_{r \psi \psi}^{\mathrm{EWSB}}=+\sum_{i j k=0}^{\infty} \frac{r_{i}}{\Lambda_{r}}\left[v \tilde{X}_{i j k}^{R L} \bar{\chi}_{1, R}^{j} \chi_{2, L}^{k}+\text { h.c. }\right]
$$

For Higgs doublets localized on the IR branes, one has $\tilde{X}_{i j k}^{R L}=2\left(Y_{5 D} / L\right)\left[1+(-1)^{i+j+k}\right] \times$ $F_{i}(L) f_{1, R}^{j}(L) f_{2, L}^{k}(L)$, where $F_{i}$ is the $r_{i}$ wavefunction. The coefficients $v \tilde{X}_{i j k}^{R L}$, after rotation to the mass eigenbasis, can be assumed to be part of the $m_{i j k}^{R L}$ of Eq. (54). The remaining contributions to the coefficients in Eq. (54) arise from the bulk terms in the 5D action, and are obtained from the overlap integrals given in Ref. [18], 14 after properly applying the unitary transformations that diagonalize the mass matrix, $m_{\mathrm{KK}}^{R L}+\tilde{m}^{R L}=U_{R} m_{\text {phys }} U_{L}^{\dagger}$, where $m_{\mathrm{KK}}^{R L}$ contains the KK masses, $\tilde{m}^{R L}$ is the EWSB mass matrix given in Eq. (55), and $m_{\text {phys }}$ is the diagonal matrix of physical masses (chosen to be real and positive).

Also, from Eq. (56) one can obtain $h-r_{i}-\psi^{j}-\psi^{k}$ contact interactions involving the Higgs field, $h$. In the mass eigenbasis, these are controlled by the dimensionless couplings

$$
X_{i j k}^{R L}=\left(U_{R}^{\dagger}\right)_{j n} \tilde{X}_{i n m}^{R L}\left(U_{L}\right)_{m k}
$$

with $X_{i j k}^{L R}=X_{i k j}^{R L}$.

For self-annihilation processes, there are also relevant terms involving two radion modes and a fermion pair. Following Ref. [18], we write these as

$$
\mathcal{L}_{\mathrm{rr} \psi \psi}=-\sum_{i_{1} i_{2}=0}^{\infty} \sum_{j k=0}^{\infty} \frac{3 r_{i_{1}} r_{i_{2}}}{2 \Lambda_{r}^{2}}\left\{g_{i_{1} i_{2} j k}^{L L} \bar{\psi}_{L}^{j} i \stackrel{\leftrightarrow}{\not} \psi_{L}^{k}+g_{i_{1} i_{2} j k}^{R R} \bar{\psi}_{R}^{j} i \stackrel{\leftrightarrow}{\not} \psi_{R}^{k}+\frac{4}{3}\left(m_{i_{1} i_{2} j k}^{R L} \bar{\psi}_{R}^{j} \psi_{L}^{k}+\text { h.c. }\right)\right\}
$$

\footnotetext{
${ }^{14}$ Some representative values were given in Tables 1 and 2 of that reference.
} 
and

$$
\mathcal{L}_{r r \psi \psi}^{\mathrm{EWSB}}=-\sum_{i_{1} i_{2}=0}^{\infty} \sum_{j k=0}^{\infty} \frac{r_{i_{1}} r_{i_{2}}}{\Lambda_{r}^{2}}\left[v \tilde{X}_{i_{1} i_{2} j k}^{R L} \bar{\chi}_{1, R}^{j} \chi_{2, L}^{k}+\text { h.c. }\right] .
$$

For a Higgs field localized on the IR branes, one has $\tilde{X}_{i_{1} i_{2} j k}^{R L}=4\left(Y_{5 D} / L\right)\left[1+(-1)^{i_{1}+i_{2}+j+k}\right] \times$ $F_{i}(L) F_{j}(L) f_{1, R}^{j}(L) f_{2, L}^{k}(L)$, where $F_{i}$ is the $r_{i}$ wavefunction. In the same way as for the single radion interactions, the coefficients $v \tilde{X}_{i_{1} i_{2} j k}^{R L}$, after rotation to the mass eigenbasis, can be assumed to be part of the $m_{i_{1} i_{2} j k}^{R L}$ above.

The Feynman rules arising from Eq. (54) were given in Ref. [18. We find it convenient to express various decay rates and cross sections involving the radion/KK-radion in terms of the dimensionless quantities

$$
G_{i_{1} \cdots i_{n} j k}^{R L} \equiv n ! \times \frac{M_{i_{1} \cdots i_{n} j k}^{R L}}{m_{j}}, \quad G_{i_{1} \cdots i_{n} j k}^{L R} \equiv n ! \times \frac{M_{i_{1} \cdots i_{n} j k}^{L R}}{m_{j}}
$$

where $m_{j}$ is the physical mass of the $j$-th KK fermion excitation, and we defined

$$
M_{i_{1} \cdots i_{n} j k}^{R L} \equiv \frac{1}{2}\left(c_{n} g_{i_{1} \cdots i_{n} j k}^{L L} m_{j}+c_{n} g_{i_{1} \cdots i_{n} j k}^{R R} m_{k}\right)-d_{n} m_{i_{1} \cdots i_{n} j k}^{R L}
$$

with $c_{n}=(-3)^{n}(2 n / 3-1) / n$ ! and $d_{n}=(-4)^{n-1} / n$ !. One also has $M_{i_{1} \cdots i_{n} j k}^{L R}=M_{i_{1} \cdots i_{n} k j}^{R L}$.

In the limit that EWSB is neglected, one has

$$
m_{i_{1} \ldots i_{n} j k}^{R L}=2 g_{i_{1} \ldots i_{n} j k}^{L L} m_{j}+2 g_{i_{1} \ldots i_{n} j k}^{R R} m_{k}+m_{i_{1} \ldots i_{n} j k}^{\text {scalar }},
$$

where $m_{i_{1} \ldots i_{n} j k}^{\text {scalar }}$ is a contribution coming from the Yukawa coupling involving the stabilizing scalar field, which is in general numerically negligible (the expression is given in [18]). In this case, the $g_{i_{1} \ldots i_{n} j k}^{L L}$ and $g_{i_{1} \ldots i_{n} j k}^{R R}$ are simply overlap integrals of $n$ radion modes and two fermion wavefunctions.

\section{B Formulas for processes involving the Radion Tower}

In the following, we use extensively the notation

$$
\epsilon_{i, j} \equiv \frac{m_{i}}{m_{j}}
$$

Also, in the following formulas, the $G_{i j k}^{R L}, G_{i j k}^{L R}, G_{i i j k}^{R L}$ and $G_{i i j k}^{L R}$ are as defined in Eqs. (59) and (60), and the $g_{i j k}^{L L}$ and $g_{i j k}^{R R}$ are as defined in Eq. (54).

\section{B.1 Self-Annihilations}

For annihilation into fermion pairs, we are only interested in the process $r^{\prime} r^{\prime} \rightarrow f f$, but we give the result for the slightly more general case $r_{i} r_{i} \rightarrow f^{k} \bar{f}^{k}$. As shown in Fig. 1, there are two types of diagrams: those involving a "heavy" $f^{j}$ exchange in the $t$ - and $u$-channels, and 
a contact interaction. The contribution due to the $t$ - and $u$-channel exchange can be written as

$$
\mathrm{v} \sigma_{r_{i} r_{i} \rightarrow f^{k} \bar{f}^{k}}^{\mathrm{t}+\mathrm{u}} \approx \sum_{j} \frac{N_{c}}{16 \pi} \frac{m_{i}^{2}}{\Lambda_{r}^{4}}\left(G_{i j k}^{\mathrm{eff}}\right)^{2}\left(1-\epsilon_{k, i}^{2}\right)^{3 / 2}
$$

where we defined

$$
\begin{aligned}
& G_{i j k}^{\mathrm{eff}} \equiv \frac{1}{\left(1+\epsilon_{j, i}^{2}-\epsilon_{k, i}^{2}\right)}\left\{2\left[\left(G_{i j k}^{R L}\right)^{2}+\left(G_{i j k}^{L R}\right)^{2}\right] \epsilon_{j, i}^{2} \epsilon_{k, i}+4 G_{i j k}^{R L} G_{i j k}^{L R} \epsilon_{j, i}^{3}\right. \\
& \left.-\left(1+\epsilon_{j, i}^{2}-\epsilon_{k, i}^{2}\right)\left(\frac{1}{2}\left[\left(g_{i j k}^{L L}\right)^{2}+\left(g_{i j k}^{R R}\right)^{2}\right] \epsilon_{k, i}+\left[2 g_{i j k}^{L L} G_{i j k}^{L R}-g_{i j k}^{L L} g_{i j k}^{R R}+2 g_{i j k}^{R R} G_{i j k}^{R L}\right] \epsilon_{j, i}\right)\right\},
\end{aligned}
$$

and $N_{c}=3\left(N_{c}=1\right)$ for quarks (leptons), while the $\epsilon$ 's are the mass ratios defined in Eq. (62), i.e. we normalize with respect to the $r_{i}$ mass. Eq. (63) holds in the deep non-relativistic limit ( $\mathrm{v}$ is the relative velocity of the initial state).

The non-relativistic contribution due to the contact interaction is

$$
\mathrm{v} \sigma_{r_{i} r_{i} \rightarrow f^{k} \bar{f}^{k}}^{\text {Contact }} \approx \frac{N_{c}}{16 \pi} \frac{m_{k}^{2}}{\Lambda_{r}^{4}} \sqrt{1-\epsilon_{k, i}^{2}}\left\{2\left[\left(G_{i i k k}^{R L}\right)^{2}+\left(G_{i i k k}^{L R}\right)^{2}\right]-\left(G_{i i k k}^{R L}+G_{i i k k}^{L R}\right)^{2} \epsilon_{k, i}^{2}\right\}
$$

while the crossed-term is given by

$$
\mathrm{v} \sigma_{r_{i} r_{i} \rightarrow f^{k} \bar{f}^{k}}^{\mathrm{Crossed}} \approx-\sum_{j} \frac{N_{c}}{8 \pi} \frac{m_{i} m_{k}}{\Lambda_{r}^{4}} G_{i j k}^{\mathrm{eff}}\left(G_{i i k k}^{R L}+G_{i i k k}^{L R}\right)\left(1-\epsilon_{k, i}^{2}\right)^{3 / 2} .
$$

Annihilation into the KK-parity even Higgs degrees of freedom (see Fig. 2), gives (see [18] for the relevant Feynman rules)

$$
\mathrm{v} \sigma_{r_{i} r_{i} \rightarrow H H} \approx \frac{N_{H} F_{i}(L)^{4}}{4 \pi} \frac{m_{i}^{2}}{\Lambda_{r}^{4}} \frac{\sqrt{1-\epsilon_{h, i}^{2}}}{\left(1+\epsilon_{H, i}^{2}-\epsilon_{h, i}^{2}\right)^{2}}\left[\epsilon_{H, i}^{2}\left(2+3 \epsilon_{h, i}^{2}\right)-11 \epsilon_{h, i}^{2}-21 \epsilon_{h, i}^{4}\right]^{2}
$$

where $\epsilon_{H, i}=m_{H} / m_{i}$ and $\epsilon_{h, i}=m_{h} / m_{i}$, with $m_{H}$ the mass of the "inert" Higgs doublet and $m_{h}$ the mass of the SM-like Higgs. $N_{H}=4$ takes into account the 4 real Higgs d.o.f. in the limit that $m_{i}$ is large. The $F_{i}(L)$ are the KK-radion wavefunctions evaluated on the IR boundaries, which are order one numbers, and in particular, $F_{r^{\prime}}(L) \approx 1$ for the first KK-radion mode.

We also quote the tree-level annihilation cross section into identical massless gauge bosons, via a massive KK gauge boson exchange (of mass $m_{j}$ ) in the t- and u-channels:

$$
\mathrm{v} \sigma_{r_{i} r_{i} \rightarrow V V} \approx \sum_{j} \frac{g_{i j 0}^{4}}{\pi} \frac{m_{i}^{2}}{\Lambda_{r}^{4}} \frac{\epsilon_{i, j}^{4}}{\left(1+\epsilon_{i, j}^{2}\right)^{2}}\left(4+2 \epsilon_{i, j}^{2}+\epsilon_{i, j}^{4}\right)
$$

where $\epsilon_{i, j}=m_{i} / m_{j}$, and $g_{i j 0}$ is the overlap integral of the wavefunctions for a radion, a massive gauge KK mode and a massless gauge boson. This dimensionless coupling is of order $1 / \sqrt{2 k_{\text {eff }} L}$. 


\section{B.2 Co-Annihilations}

For coannihilations with the NLKP, involving gluons in the final state, we are interested in the process $r^{\prime} t^{\prime} \rightarrow g t$, but we give the result for the more general case $r_{i} t^{j} \rightarrow g t^{k}$. Including the $s$ - and $t$-channel diagrams, as well as a quartic contact interaction that is required by gauge invariance (see Ref. [18]), we have:

$$
\mathrm{v} \sigma_{r_{i} t^{j} \rightarrow g t^{k}} \approx \frac{\left(N_{c}^{2}-1\right) \alpha_{s}}{16 N_{c} \Lambda_{r}^{2}}\left[\left(G_{i j k}^{R L}\right)^{2}+\left(G_{i j k}^{L R}\right)^{2}\right]\left(\frac{1}{1+\epsilon_{j, i}}\right)\left[1-\left(\frac{\epsilon_{k, i}}{1+\epsilon_{j, i}}\right)^{2}\right]
$$

which holds in the ultra non-relativistic limit ( $\mathrm{v}$ is the relative velocity of the initial state). The $\epsilon$ 's are the mass ratios defined in Eq. (62), i.e. we normalize with respect to the $r_{i}$ mass.

There are also channels with a Higgs in the final state. We are interested in $r^{\prime} t^{\prime} \rightarrow h t$, but we quote the more general case (in the ultra non-relativistic limit, and neglecting a subdominant diagram with a $s$-channel $\left.t^{k}\right)$ :

$$
\begin{aligned}
& \mathrm{v} \sigma_{r_{i} t^{j} \rightarrow h t^{k}} \approx \frac{1}{64 \pi \Lambda_{r}^{2}} \frac{\sqrt{\left[\left(1+\epsilon_{j, i}+\epsilon_{h, i}\right)^{2}-\epsilon_{k, i}^{2}\right]\left[\left(1+\epsilon_{j, i}-\epsilon_{h, i}\right)^{2}-\epsilon_{k, i}^{2}\right]}}{\left(1+\epsilon_{j, i}\right)^{3}} \times \\
&\left\{\left[\left(X_{i j k}^{R L}\right)^{2}+\left(X_{i j k}^{L R}\right)^{2}\right]\left[\left(1+\epsilon_{j, i}\right)^{2}+\epsilon_{k, i}^{2}-\epsilon_{h, i}^{2}\right]+4 X_{i j k}^{R L} X_{i j k}^{L R} \epsilon_{k, i}\left(1+\epsilon_{j, i}\right)\right\},
\end{aligned}
$$

where $X_{i j k}^{R L}$ and $X_{i j k}^{L R}$ were defined in Eq. (57). The $\epsilon$ 's are the mass ratios defined in Eq. (62), and we also defined $\epsilon_{h, i} \equiv m_{h} / m_{i}$, where $m_{h}$ is the Higgs mass (i.e. we normalize all the masses to the $r_{i}$ mass).

\section{B.3 $f^{j}$ Decays}

The production of $r_{i}$ in $f^{j}$ decays is governed by

$$
\begin{aligned}
\Gamma_{f^{j} \rightarrow r_{i} f^{k}}^{\mathrm{CM}}= & \frac{m_{j}^{3}}{16 \pi \Lambda_{r}^{2}} \sqrt{\left[1-\left(\epsilon_{k, j}+\epsilon_{i, j}\right)^{2}\right]\left[1-\left(\epsilon_{k, j}-\epsilon_{i, j}\right)^{2}\right]} \\
& \times\left\{\frac{1}{2}\left[\left(G_{i j k}^{R L}\right)^{2}+\left(G_{i j k}^{L R}\right)^{2}\right]\left(1+\epsilon_{k, j}^{2}-\epsilon_{i, j}^{2}\right)+2 G_{i j k}^{R L} G_{i j k}^{L R} \epsilon_{k, j}\right\},
\end{aligned}
$$

where the $\epsilon$ 's are the mass ratios defined in Eq. 62, i.e. we normalize with respect to the mass of the decaying particle.

\section{B.4 $V_{\mu}^{j}$ Decays}

The production of $r_{i}$ in $V_{\mu}^{j}$ decays, when the final gauge boson has mass $m_{k} \neq 0$, is governed by

$$
\Gamma_{V_{\mu}^{j} \rightarrow V_{\mu}^{k} r_{i}}^{\mathrm{CM}} \approx \frac{\left(G_{i j k}^{\mathrm{eff}, \mathrm{V}}\right)^{2} m_{j}^{5}}{192 \pi m_{k}^{2} \Lambda_{r}^{2}} \sqrt{\left[1-\left(\epsilon_{k, j}+\epsilon_{i, j}\right)^{2}\right]\left[1-\left(\epsilon_{k, j}-\epsilon_{i, j}\right)^{2}\right]}\left[\left(1+\epsilon_{k, j}^{2}-\epsilon_{i, j}^{2}\right)^{2}+8 \epsilon_{k, j}^{2}\right]
$$


where

$$
G_{i j k}^{\mathrm{eff}, \mathrm{V}} \equiv 4 g_{i j k}^{m} \epsilon_{k, j}+g_{i j k}\left(1+\epsilon_{k, j}^{2}-\epsilon_{i, j}^{2}\right)
$$

and

$$
g_{i j k}=\frac{1}{2 L} \int_{-L}^{L} d y F_{i} f_{V}^{j} f_{V}^{k}, \quad g_{i j k}^{m}=\frac{1}{2 L} \int_{-L}^{L} d y e^{-2 A} F_{i} f_{5}^{j} f_{5}^{k}
$$

with $f_{5}^{j}(y)$ the wavefunction corresponding to $V_{5}^{j}$ (which is related to the $V_{\mu}^{j}$ wavefunction by $\left.f_{5}^{j}=\partial_{y} f_{V}^{j} / m_{j}\right)$.

When $m_{k}=0$, we have instead

$$
\Gamma_{V_{\mu}^{j} \rightarrow V_{\mu}^{0} r_{i}}^{\mathrm{CM}} \approx \frac{g_{i j k}^{2} m_{j}^{3}}{16 \pi \Lambda_{r}^{2}}\left(1-\epsilon_{i, j}\right)^{3}
$$

where $g_{i j k}$ is suppressed by order $1 / \sqrt{2 k_{\text {eff }} L}$ due to the massless wavefunction.

\section{B.5 KK-parity odd Higgs Decays}

The states in the KK-parity odd Higgs doublet can decay into $r^{\prime}$ plus a KK-parity even Higgs.

Parameterizing the KK-parity odd and even Higgs doublets as $H_{-}=\left\{H^{+}, \frac{1}{\sqrt{2}}\left(h_{-}+i a\right)\right\}$ and $H_{+}=\left\{G^{+}, \tilde{v}+\frac{1}{\sqrt{2}}\left(h_{+}+i G^{0}\right)\right\}$, respectively, where $\tilde{v}=174 \mathrm{GeV}, h_{+}$is the SM-like Higgs and $G^{0}, G^{ \pm}$are the would-be Nambu-Goldstone bosons, we have

$$
\begin{aligned}
\Gamma_{h_{-} \rightarrow h_{+} r^{\prime}}^{\mathrm{CM}=} & \frac{F_{r^{\prime}}(L)^{2} m_{h_{-}}^{3}}{16 \pi \Lambda_{r}^{2}}\left(1-7 \epsilon_{h_{+}, h_{-}}^{2}-\epsilon_{r^{\prime}, h_{-}}^{2}\right)^{2} \\
& \times \sqrt{\left[1-\left(\epsilon_{h_{+}, h_{-}}+\epsilon_{r^{\prime}, h_{-}}\right)^{2}\right]\left[1-\left(\epsilon_{h_{+}, h_{-}}-\epsilon_{r^{\prime}, h_{-}}\right)^{2}\right]}
\end{aligned}
$$

for the CP-even Higgs, and

$$
\Gamma_{a \rightarrow G^{0} r^{\prime}}^{\mathrm{CM}}=\frac{F_{r^{\prime}}(L)^{2} m_{a}^{3}}{16 \pi \Lambda_{r}^{2}}\left(1-\epsilon_{r^{\prime}, a}^{2}\right)^{3}, \quad \Gamma_{H^{ \pm} \rightarrow G^{ \pm} r^{\prime}}^{\mathrm{CM}}=\frac{F_{r^{\prime}}(L)^{2} m_{H^{ \pm}}^{3}}{16 \pi \Lambda_{r}^{2}}\left(1-\epsilon_{r^{\prime}, H^{ \pm}}^{2}\right)^{3}
$$

for the CP-odd and charged Higgses. Here $F_{r^{\prime}}(L) \approx 1$.

\section{B.6 Production of $r_{i}$ through Scattering}

The $r_{i}$ production cross section (times flux) via the process $g q^{j} \rightarrow r_{i} q^{k}$ (which includes $s$ and $t$-channel diagrams, as well as contact interactions required by gauge invariance) can be written as

$$
\begin{aligned}
F \sigma_{g q^{j} \rightarrow r_{i} q^{k}}= & \frac{\alpha_{s}}{64 N_{c}} \frac{m_{j}^{2}}{\Lambda_{r}^{2}} \frac{\theta\left(z, \Delta \epsilon, \epsilon_{k, j}\right)}{(1-z)^{2}} \times \\
& \left\{\left[\left(G_{i j k}^{R L}\right)^{2}+\left(G_{i j k}^{L R}\right)^{2}\right] \mathcal{F}_{1}\left(z, \Delta \epsilon, \epsilon_{k, j}\right)+G_{i j k}^{R L} G_{i j k}^{L R} \mathcal{F}_{2}\left(z, \Delta \epsilon, \epsilon_{k, j}\right)\right\},
\end{aligned}
$$


where

$$
\begin{aligned}
\theta\left(z, \Delta \epsilon, \epsilon_{k, j}\right)= & \sqrt{(1+\Delta \epsilon z)^{2}-4 \epsilon_{k, j}^{2} z}, \\
\mathcal{F}_{1}\left(z, \Delta \epsilon, \epsilon_{k, j}\right)= & 4\left[1+2 \Delta \epsilon z+\left(1+2 \Delta \epsilon+2 \Delta \epsilon^{2}\right) z^{2}\right] \frac{\tan ^{-1}\left[\frac{\theta\left(z, \Delta \epsilon, \epsilon_{k, j}\right)}{1+\Delta \epsilon z}\right]}{\theta\left(z, \Delta \epsilon, \epsilon_{k, j}\right)} \\
& -\left[3+(2+7 \Delta \epsilon) z+(3+2 \Delta \epsilon) z^{2}-\Delta \epsilon z^{3}\right], \\
\mathcal{F}_{2}\left(z, \Delta \epsilon, \epsilon_{k, j}\right)= & 32 \epsilon_{k, j} z\left[(1+\Delta \epsilon z) \frac{\tan ^{-1}\left[\frac{\theta\left(z, \Delta \epsilon, \epsilon_{k, j}\right)}{1+\Delta \epsilon z}\right]}{\theta\left(z, \Delta \epsilon, \epsilon_{k, j}\right)}-1\right] .
\end{aligned}
$$

Here $z=m_{j}^{2} / s$, with $\sqrt{s}$ the CM energy, $\Delta \epsilon \equiv \epsilon_{k, j}^{2}-\epsilon_{i, j}^{2}$, and the $\epsilon$ 's are the mass ratios defined in Eq. (62), i.e. we normalize with respect to the incident $t^{j}$ mass. The flux factor is given in the CM frame by $F=\sqrt{s}|\vec{p}|$, where $|\vec{p}|$ is the momentum of any of the initial state particles, and replaces the relative velocity appropriate for the non-relativistic scattering of massive particles.

The singularity at $z=1$ in Eq. (78) arises from the $s$-channel diagram, and is regularized by the $q^{j}$ width, $\Gamma_{j}$. For instance, in the non-relativistic regime, $z \approx 1$, we have

$$
\begin{aligned}
F \sigma_{g q^{j} \rightarrow r_{i} q^{k}} \approx & \frac{\alpha_{s}}{8 N_{c}} \frac{m_{j}^{2}}{\Lambda_{r}^{2}} \frac{m_{j}^{4}}{\left(s-m_{j}^{2}\right)^{2}+m_{j}^{2} \Gamma_{j}^{2}}\left[(1+\Delta \epsilon) \frac{\tan ^{-1}\left[\frac{\theta\left(1, \Delta \epsilon, \epsilon_{k, j}\right)}{1+\Delta \epsilon}\right]}{\theta\left(1, \Delta \epsilon, \epsilon_{k, j}\right)}-1\right] \\
& \times \theta\left(1, \Delta \epsilon, \epsilon_{k, j}\right)\left\{\left[\left(G_{i j k}^{R L}\right)^{2}+\left(G_{i j k}^{L R}\right)^{2}\right](1+\Delta \epsilon)+4 G_{i j k}^{R L} G_{i j k}^{L R} \epsilon_{k, j}\right\} .
\end{aligned}
$$

In the ultra-relativistic regime, Eq. (78) reduces to

$$
F \sigma_{g q^{j} \rightarrow r_{i} q^{k}} \approx \frac{\alpha_{s}}{64 N_{c}} \frac{m_{j}^{2}}{\Lambda_{r}^{2}}\left[\left(G_{i j k}^{R L}\right)^{2}+\left(G_{i j k}^{L R}\right)^{2}\right]\left[2 \log \left(\frac{s}{m_{k}^{2}}\right)-3\right]
$$

\section{B.7 Radion Decays}

We also summarize the partial decay widths for the radion (KK-parity even) decays into fermion pairs, massive and massless gauge bosons, and the CP-even Higgs. These are used in Section 6.2. For the decays into fermion pairs, we have

$$
\Gamma_{r \rightarrow f \bar{f}}=\frac{m_{r} m_{f}^{2}}{16 \pi \Lambda_{r}^{2}} \sqrt{1-4 \epsilon_{f, r}^{2}}\left\{\left[\left(G_{000}^{R L}\right)^{2}+\left(G_{000}^{L R}\right)^{2}\right]\left(1-2 \epsilon_{f, r}^{2}\right)-4 G_{000}^{R L} G_{000}^{L R} \epsilon_{f, r}^{2}\right\}
$$

where $\epsilon_{f, r}=m_{f} / m_{r}$, and a color factor $N_{c}$ should be included for quarks.

For the decays into (indistinguishable) massive gauge bosons, we have

$$
\Gamma_{r \rightarrow V V}^{\text {massive }} \approx \frac{F_{0}(L)^{2} m_{r}^{3}}{32 \pi \Lambda_{r}^{2}} \sqrt{1-4 \epsilon_{V, r}^{2}}\left(1-4 \epsilon_{V, r}^{2}+12 \epsilon_{V, r}^{4}\right)
$$


where $\epsilon_{V, r}=m_{V} / m_{r}$, and $F_{0}(L) \approx 1$ is the wavefunction of the radion evaluated on the IR brane. Here we have assumed that the Higgs (hence EWSB) is IR-localized, and neglected a subdominant contribution from the bulk, present even for massless gauge bosons. The decay rate into massless gauge bosons is

$$
\Gamma_{r \rightarrow V V}^{\text {massless }}=\frac{g_{000}^{2} m_{r}^{3}}{16 \pi \Lambda_{r}^{2}},
$$

where $g_{000} \approx 1 /\left(2 k_{\text {eff }} L\right)$ is the overlap integral for a radion and two massless gauge boson wavefunctions (the latter being flat).

For the decays into the CP-even Higgs, $h_{+}$, we have

$$
\Gamma_{r \rightarrow h h}=\frac{F_{0}(L)^{2} m_{r}^{3}}{32 \pi \Lambda_{r}^{2}} \sqrt{1-4 \epsilon_{h, r}^{2}}\left(1+2 \epsilon_{h, r}^{2}\right)^{2},
$$

where $\epsilon_{h, r}=m_{h} / m_{r}$, and a factor of $1 / 2$ for identical particles has been included. A similar expression applies for the decay into the KK-parity odd Higgses, provided these channels are kinematically open.

\section{References}

[1] H. C. Cheng and I. Low, JHEP 0309, 051 (2003) arXiv:hep-ph/0308199.

[2] T. Appelquist, H. -C. Cheng, B. A. Dobrescu, Phys. Rev. D64, 035002 (2001). hep$\mathrm{ph} / 0012100$.

[3] H. C. Cheng, K. T. Matchev and M. Schmaltz, Phys. Rev. D 66, 036005 (2002) arXiv:hep-ph/0204342].

[4] B. A. Dobrescu and E. Pontón, JHEP 0403, 071 (2004) arXiv:hep-th/0401032;

G. Burdman, B. A. Dobrescu and E. Pontón, JHEP 0602, 033 (2006) arXiv:hepph/0506334.

[5] G. Servant, T. M. P. Tait, Nucl. Phys. B650, 391-419 (2003). hep-ph/0206071.

[6] G. Servant, T. M. P. Tait, New J. Phys. 4, 99 (2002). hep-ph/0209262].

[7] G. Bertone, D. Hooper, J. Silk, Phys. Rept. 405, 279-390 (2005). hep-ph/0404175.

[8] L. Randall and R. Sundrum, Phys. Rev. Lett. 83, 3370 (1999) arXiv:hep-ph/9905221].

[9] E. Pontón and L. Randall, JHEP 0904, 080 (2009) [arXiv:0811.1029 [hep-ph]].

[10] K. Agashe, G. Servant, Phys. Rev. Lett. 93, 231805 (2004). hep-ph/0403143.

[11] G. Panico, E. Pontón, J. Santiago and M. Serone, Phys. Rev. D 77, 115012 (2008) arXiv:0801.1645 [hep-ph]]. 
[12] M. Carena, A. D. Medina, N. R. Shah and C. E. M. Wagner, Phys. Rev. D 79, 096010 (2009) arXiv:0901.0609 [hep-ph]].

[13] A. R. Frey, R. J. Danos and J. M. Cline, JHEP 0911, 102 (2009) arXiv:0908.1387 [hep-th]].

[14] K. Agashe, K. Blum, S. J. Lee and G. Perez, Phys. Rev. D 81, 075012 (2010) arXiv:0912.3070 [hep-ph]].

[15] T. Gherghetta and B. von Harling, JHEP 1004, 039 (2010) arXiv:1002.2967[hep-ph]].

[16] K. Agashe, A. Falkowski, I. Low and G. Servant, JHEP 0804, 027 (2008) arXiv:0712.2455[hep-ph]].

[17] W. D. Goldberger and M. B. Wise, Phys. Rev. Lett. 83, 4922 (1999) arXiv:hepph/9907447.

[18] A. D. Medina and E. Pontón, arXiv:1012.5298 [hep-ph].

[19] K. L. McDonald, Phys. Rev. D 80, 024038 (2009) arXiv:0905.3006 [hep-ph]].

[20] Y. Grossman and M. Neubert, Phys. Lett. B 474, 361 (2000) arXiv:hep-ph/9912408.

[21] T. Gherghetta and A. Pomarol, Nucl. Phys. B 586 (2000) 141 arXiv:hep-ph/0003129.

[22] C. Delaunay, O. Gedalia, S. J. Lee et al., arXiv:1007.0243 [hep-ph]].

[23] C. Delaunay, O. Gedalia, S. J. Lee et al., arXiv:1101.2902 [hep-ph]].

[24] P. Creminelli, A. Nicolis and R. Rattazzi, JHEP 0203, 051 (2002) arXiv:hepth/0107141].

[25] L. Randall and G. Servant, JHEP 0705, 054 (2007) arXiv:hep-ph/0607158.

[26] J. Kaplan, P. C. Schuster and N. Toro, arXiv:hep-ph/0609012.

[27] G. Nardini, M. Quirós and A. Wulzer, JHEP 0709, 077 (2007) arXiv:0706.3388 [hep$\mathrm{ph}]$.

[28] B. Hassanain, J. March-Russell and M. Schvellinger, JHEP 0710, 089 (2007) arXiv:0708.2060 [hep-th]].

[29] T. Konstandin, G. Nardini and M. Quiros, Phys. Rev. D 82, 083513 (2010) arXiv:1007.1468 [hep-ph]].

[30] T. Konstandin and G. Servant, arXiv:1104.4791 [hep-ph].

[31] J. M. Maldacena, Adv. Theor. Math. Phys. 2, 231 (1998) [Int. J. Theor. Phys. 38, 1113 (1999)] arXiv:hep-th/9711200.

[32] E. Witten, Adv. Theor. Math. Phys. 2, 505 (1998) arXiv:hep-th/9803131]. 
[33] E. W. Kolb and M. S. Turner, Redwood City, USA: Addison-Wesley (1990) $547 p$. (Frontiers in physics, 69).

[34] C. Csaki, M. Graesser, L. Randall and J. Terning, Phys. Rev. D 62, 045015 (2000) arXiv:hep-ph/9911406.

[35] G. F. Giudice, R. Rattazzi and J. D. Wells, Nucl. Phys. B 595, 250 (2001) arXiv:hepph/0002178.

[36] Z. Chacko, M. A. Luty and E. Pontón, JHEP 0007, 036 (2000) arXiv:hep-ph/9909248.

[37] K. Griest and D. Seckel, Phys. Rev. D 43 (1991) 3191.

[38] E. Komatsu et al., arXiv:1001.4538 [astro-ph.CO].

[39] E. W. Kolb, G. Servant, T. M. P. Tait, JCAP 0307, 008 (2003). hep-ph/0306159.

[40] T. Moroi, H. Murayama and M. Yamaguchi, Phys. Lett. B 303, 289 (1993).

[41] J. L. Feng, A. Rajaraman and F. Takayama, Phys. Rev. Lett. 91, 011302 (2003) arXiv:hep-ph/0302215.

[42] J. L. Feng, A. Rajaraman and F. Takayama, Phys. Rev. D 68, 063504 (2003) arXiv:hep-ph/0306024.

[43] P. Gondolo, G. Gelmini, Nucl. Phys. B360, 145-179 (1991).

[44] J. L. Feng, S. f. Su and F. Takayama, Phys. Rev. D 70, 063514 (2004) arXiv:hepph/0404198.

[45] K. Kohri, Phys. Rev. D 64, 043515 (2001) arXiv:astro-ph/0103411.

[46] M. Kawasaki, K. Kohri and T. Moroi, Phys. Lett. B 625, 7 (2005) arXiv:astroph/0402490.

[47] C. P. Burgess, M. Pospelov and T. ter Veldhuis, Nucl. Phys. B 619, 709 (2001) arXiv:hep-ph/0011335.

[48] E. Aprile et al. [XENON100 Collaboration], arXiv:1104.2549 [astro-ph.CO].

[49] F. Halzen, D. Hooper, New J. Phys. 11, 105019 (2009). arXiv:0910.4513 [astroph.HE]].

[50] C. Csaki, J. Hubisz and S. J. Lee, Phys. Rev. D 76, 125015 (2007) arXiv:0705.3844 [hep-ph]].

[51] J. F. Navarro, C. S. Frenk and S. D. M. White, Astrophys. J. 462, 563 (1996) arXiv:astro-ph/9508025].

[52] F. Donato et al., arXiv:0904.4054 [astro-ph.CO]. 
[53] P. Salucci, F. Nesti, G. Gentile and C. F. Martins, Astron. Astrophys. 523, A83 (2010) arXiv:1003.3101 [astro-ph.GA]].

[54] G. Bertone, C. B. Jackson, G. Shaughnessy, T. M. P. Tait, A. Vallinotto, [arXiv:1009.5107 [astro-ph.HE]].

[55] G. Belanger, F. Boudjema, P. Brun, A. Pukhov, S. Rosier-Lees, P. Salati, A. Semenov, Comput. Phys. Commun. 182, 842-856 (2011). [arXiv:1004.1092 [hep-ph]].

[56] H. -C. Cheng, J. L. Feng, K. T. Matchev, Phys. Rev. Lett. 89, 211301 (2002). hep$\mathrm{ph} / 0207125$.

[57] L. Bergstrom, T. Bringmann, M. Eriksson and M. Gustafsson, Phys. Rev. Lett. 94, 131301 (2005) arXiv:astro-ph/0410359.

[58] L. Bergstrom, T. Bringmann, M. Eriksson and M. Gustafsson, JCAP 0504, 004 (2005) arXiv:hep-ph/0412001.

[59] H. Davoudiasl, S. Gopalakrishna, E. Pontón and J. Santiago, New J. Phys. 12, 075011 (2010) arXiv:0908.1968 [hep-ph]].

[60] H. -C. Cheng, K. T. Matchev, M. Schmaltz, Phys. Rev. D66, 056006 (2002). hep$\mathrm{ph} / 0205314$.

[61] G. Burdman, B. A. Dobrescu, E. Ponton, Phys. Rev. D74, 075008 (2006). hepph/0601186.

[62] E. Pontón, L. Wang, JHEP 0611, 018 (2006). hep-ph/0512304.

[63] G. Azuelos et al., Eur. Phys. J. C 39S2, 13 (2005) arXiv:hep-ph/0402037.

[64] J. A. Aguilar-Saavedra, Phys. Lett. B 625, 234 (2005) [Erratum-ibid. B 633, 792 (2006)] [arXiv:hep-ph/0506187]; J. A. Aguilar-Saavedra, PoS TOP2006, 003 (2006) arXiv:hep-ph/0603199].

[65] P. Meade and M. Reece, Phys. Rev. D 74, 015010 (2006) arXiv:hep-ph/0601124.

[66] S. Bae, P. Ko, H. S. Lee and J. Lee, Phys. Lett. B 487, 299 (2000) arXiv:hep$\mathrm{ph} / 0002224$. 